\title{
ON TRIPLES OF IDEAL CHAMBERS IN $A_{2}$-BUILDINGS
}

\author{
ANNE PARREAU
}

\begin{abstract}
We investigate the geometry in a real Euclidean building $X$ of type $A_{2}$ of some simple configurations in the associated projective plane at infinity $\mathbb{P}$, seen as ideal configurations in $X$, and relate it with the projective invariants (from the cross ratio on $\mathbb{P}$ ). In particular we establish a geometric classification of generic triples of ideal chambers of $X$ and relate it with the triple ratio of triples of flags.
\end{abstract}

\section{INTRODUCTION}

The triples of objects in the boundaries of geometric spaces $X$ are basic tools, for example in the study of surface group representations. For instance, in the case where $X=\mathbb{H}^{2}$, ideal triples of points may be used to define the notion of Euler class [Gol80], and Penner-Thurston shear coordinates on the Teichmüller space. In the case where $X=\mathbb{H}_{\mathbb{C}}^{2}$, the ideal triples are classified by Cartan's angular invariant, see for example [Gol99, §7.1], and they may be for instance used to define Toledo's invariant and maximal representations, see [Tol89]. See for instance [CN06, BIW10] for generalization to higher rank Hermitian symmetric spaces $X$, and triples in their Shilov boundary.

For higher rank symmetric spaces $X$ of type $A_{N-1}$, corresponding to the group $\mathrm{PGL}_{N}(\mathbb{R})$, ideal configurations in $X$ may be seen as configurations in the projective space $\mathbb{P}=\mathbb{P}\left(\mathbb{R}^{N}\right)$. In particular, ideal chambers of $X$ correspond to complete flags in $\mathbb{P}$, and opposite pairs of flags (or generic $N$-tuples of points) in $\mathbb{P}$ correspond to maximal flats in $X$. This is still true in the non-Archimedean setting, i.e. replacing $\mathbb{R}$ by an ultrametric valued field $\mathbb{K}$, in which case $X$ is a Euclidean building of type $A_{N-1}$.

Configurations in projective spaces $\mathbb{P}\left(\mathbb{R}^{N}\right)$ have been widely studied and used. In particular, triples of flags in $\mathbb{P}\left(\mathbb{R}^{N}\right)$ and their classical invariants (the triple ratio for $N=3$ ), are the basic building blocks used by Fock and Goncharov to define generalized shearing coordinates for higher Teichmüller space, parametrizing positive representations of punctured surface groups in $G=\mathrm{SL}_{N}(\mathbb{R})$, see [FG06]. But the geometric properties in the symmetric space or Euclidean building $X$ of these configurations remain mysterious.

In this article, we investigate the geometry of some simple ideal configurations in a (not necessarily discrete) Euclidean building $X$ of type $A_{2}$, mainly the generic triples of ideal chambers, and the relationship with their projective geometry in the projective plane $\mathbb{P}$. Our first motivation is to use it to study actions of surface groups

2010 Mathematics Subject Classification. 51E24,20E42.

Key words and phrases. Euclidean buildings, projective planes, ideal configurations, triple ratio. 
on Euclidean buildings of type $A_{2}$, and degenerations of Hitchin representations in $\mathrm{SL}_{3}(\mathbb{R})($ see $[\operatorname{Par} 15])$.

The main result is a classification of ideal triples of chambers by the geometry of the five naturally associated flats in $X$, in relation with their triple ratio as triples of flags in $\mathbb{P}$. In the case where $X$ is a real tree (e.g. a Euclidean building of type $A_{1}$ ), any generic ideal triple bounds a tripod in $X$, that is a convex subset consisting of union of three rays from a point $x \in X$ (the center of the tripod). This is no longer the case in general in higher rank buildings like $A_{2}$-buildings, and many types of configurations are possible. A special case was studied by A. Balser, who established a characterization of triples of points in $\partial_{\infty} X$ bounding a tripod in $X$ [Bal08], and used it to study convex rank 1 subsets in $A_{2}$-buildings. We give here a complete and precise description.

We now get into more details. Let $X$ be a real Euclidean building of (vectorial) type $A_{2}$, i.e. with model flat the Euclidean plane

$$
\mathbb{A}=\left\{\lambda=\left(\lambda_{1}, \lambda_{2}, \lambda_{3}\right) \in \mathbb{R}^{3} / \sum_{i} \lambda_{i}=0\right\}
$$

endowed with the finite reflection group $W=\mathfrak{S}_{3}$ acting by permutation of the coordinates. Note that $X$ is not necessarily discrete (simplicial) nor locally compact, and possibly exotic.

The boundary at infinity of $X$ may be identified with the incidence graph of an associated projective plane $\mathbb{P}=\mathbb{P}_{\infty}(X)$, equipped with an $\mathbb{R}$-valued additive cross ratio $\boldsymbol{\beta}$ (called a projective valuation in [Tit86]) defined on quadruples of pairwise distinct collinear points in $\mathbb{P}$ [Tit86]. In the algebraic case, i.e. when $X$ is the Bruhat-Tits building $X\left(\mathbb{K}^{3}\right)$ associated with the group $\mathrm{PGL}\left(\mathbb{K}^{3}\right)$ for some ultrametric field $\mathbb{K}$, the projective plane $\mathbb{P}$ is $\mathbb{P}\left(\mathbb{K}^{3}\right)$ and $\boldsymbol{\beta}$ is the logarithm

$$
\boldsymbol{\beta}=\log |\mathbf{b}|
$$

of the absolute value of the usual $\mathbb{K}$-valued cross ratio $\mathbf{b}$ on $\mathbb{P}\left(\mathbb{K}^{3}\right)$, where conventions on cross ratios are taken such that

$$
\mathbf{b}(\infty,-1,0, Z)=Z
$$

in $\mathbb{P}^{1} \mathbb{K}=\mathbb{K} \cup\{\infty\}$ (following [FG06]). We will then call $\boldsymbol{\beta}$ the geometric cross ratio and $\mathbf{b}$ the algebraic cross ratio to distinguish between them.

We now turn to ideal triples of chambers. Let $T=\left(F_{1}, F_{2}, F_{3}\right)$ be a triple of chambers at infinity of $X$. We denote by $F_{i}=\left(p_{i}, D_{i}\right)$ the corresponding flag of $\mathbb{P}$, with $p_{i}$ the point and $D_{i}$ the line. The set $\{1,2,3\}$ of indices will be canonically identified with $\mathbb{Z} / 3 \mathbb{Z}$. A triple $T=\left(F_{1}, F_{2}, F_{3}\right)$ will be called generic if the flags $\left(F_{i}\right)_{i}$ are pairwise opposite, the points $\left(p_{i}\right)_{i}$ are not collinear and the lines $\left(D_{i}\right)_{i}$ are not concurrent.

In the algebraic case $\mathbb{P}=\mathbb{P}\left(\mathbb{K}^{3}\right)$ generic triples of flags $T=\left(F_{1}, F_{2}, F_{3}\right)$ are classified by one $\mathbb{K}$-valued invariant, the (algebraic) triple ratio (see for example [FG06, §9.4]), that may be defined by:

$$
\operatorname{Tri}\left(F_{1}, F_{2}, F_{3}\right)=\mathbf{b}\left(D_{1}, p_{1} p_{2}, p_{1} p_{23}, p_{1} p_{3}\right)
$$

where $p_{i j}=D_{i} \cap D_{j}$. We recall that it is invariant under cyclic permutations of $T$, and that reversing the order inverses the algebraic triple ratio: $\operatorname{Tri}(\bar{T})=\operatorname{Tri}(T)^{-1}$, where $\bar{T}=\left(F_{3}, F_{2}, F_{1}\right)$. 
In the general case, we introduce an invariant for generic triples of flags in $\mathbb{P}$, analoguous to the algebraic triple ratio: the geometric triple ratio, which still make sense when the building $X$ is exotic (non algebraic), whereas the algebraic triple ratio is not defined anymore. We define it as the triple

$$
\operatorname{tri}(T)=\left(\operatorname{tri}_{m}(T)\right)_{m=1,2,3}
$$

of the following cross ratios in $\mathbb{P}$, which are the cross ratios obtained from the four lines $D_{1}, p_{1} p_{2}, p_{1} p_{23}, p_{1} p_{3}$ by cyclic permutation of the three last one:

$$
\begin{aligned}
& \operatorname{tri}_{1}\left(F_{1}, F_{2}, F_{3}\right)=\boldsymbol{\beta}\left(D_{1}, p_{1} p_{2}, p_{1} p_{23}, p_{1} p_{3}\right) \\
& \operatorname{tri}_{2}\left(F_{1}, F_{2}, F_{3}\right)=\boldsymbol{\beta}\left(D_{1}, p_{1} p_{3}, p_{1} p_{2}, p_{1} p_{23}\right) \\
& \operatorname{tri}_{3}\left(F_{1}, F_{2}, F_{3}\right)=\boldsymbol{\beta}\left(D_{1}, p_{1} p_{23}, p_{1} p_{3}, p_{1} p_{2}\right)
\end{aligned} .
$$

To simplify notations, we denote from now on

$$
z_{m}=\operatorname{tri}_{m}(T) \text { and } z=\left(z_{1}, z_{2}, z_{3}\right)=\operatorname{tri}(T)
$$

In the algebraic case, we have $\mathbb{P}=\mathbb{P}\left(\mathbb{K}^{3}\right)$ and the geometric triple ratio is obtained from the algebraic cross ratio $Z=\operatorname{Tri}(T) \in \mathbb{K}$ by:

$$
\begin{aligned}
& z_{1}=\log |Z| \\
& z_{2}=\log \left|\frac{1}{1+Z}\right|=-\log |1+Z| \\
& z_{3}=\log \left|1+Z^{-1}\right| .
\end{aligned}
$$

The geometric triple ratio $z$ enjoys the following properties. It is invariant by cyclic permutations of the flags, and changed to $\left(-z_{1},-z_{3},-z_{2}\right)$ by permutations reversing the cyclic order. We also have $z_{1}+z_{2}+z_{3}=0$, and the stronger following property: for all $m \in \mathbb{Z} / 3 \mathbb{Z}$, if $z_{m}>0$ then $z_{m-1}=0$ and $z_{m+1}=-z_{m}<0$. Note that the three natural cases: $z \in \mathbb{R}_{+}(0,1,-1), z \in \mathbb{R}_{+}(-1,0,1)$, and $z \in \mathbb{R}_{+}(1,-1,0)$ subdivide in two types, as the case $z_{1}=0$ is invariant under reversing the order of $T$, whereas the two other cases are exchanged.

We now turn to the geometry inside the Euclidean building $X$. A generic triple $T=\left(F_{1}, F_{2}, F_{3}\right)$ of ideal chambers defines five natural flats in $X$ : the three flats $A_{i j}=A\left(F_{i}, F_{j}\right)$ containing the opposite chambers $F_{i}$ and $F_{j}$ in their boundaries, the flat $A_{p}=A\left(p_{1}, p_{2}, p_{3}\right)$ containing the triple of ideal singular points $\left(p_{1}, p_{2}, p_{3}\right)$ in its boundary, and the similarly defined flat $A_{D}=A\left(D_{1}, D_{2}, D_{3}\right)$. We will show that there are also six particular points in $X$ naturally associated with the triple $T$, that may be defined as the orthogonal projections $y_{i}$ and $y_{i}^{*}$ (which happen to be unique) of $p_{i}$ and $D_{i}$ on the flat $A_{j k}$ where $j=i+1$ and $k=i+2$.

We say that $\left(F_{1}, F_{2}, F_{3}\right)$ is of type "tripod" if there exists a tripod in $X$ joining the three (middle points of the) ideal chambers $\left(F_{1}, F_{2}, F_{3}\right)$. The set of centers of such tripods is the intersection $I$ of the three flats $A_{i j}$.

We show that either the three flats $A_{i j}$ have nonempty intersection, i.e. $\left(F_{1}, F_{2}, F_{3}\right)$ is of type "tripod", or the two flats $A_{p}$ and $A_{D}$ have non empty intersection $\Delta$, which is then a flat singular triangle (that is, a triangle in $\mathbb{A}$ with singular sides) (we then say that $\left(F_{1}, F_{2}, F_{3}\right)$ is of type "flat"). The two following results describe more precisely the two possible types, and relate them with the points $y_{i}, y_{i}^{*}$ and the geometric triple ratio $z$. We denote by $\mathfrak{C}=\left\{\lambda \in \mathbb{A} / \lambda_{1}>\lambda_{2}>\lambda_{3}\right\}$ the model Weyl chamber of $\mathbb{A}$ and we use the corresponding simple roots coordinates on $\mathbb{A}$, that is $\lambda=\left(\lambda_{1}-\lambda_{2}, \lambda_{2}-\lambda_{3}\right)$. 
Theorem 0.1 (Type "tripod"). The intersection $I=A_{12} \cap A_{23} \cap A_{31}$ is nonempty if and only if $z_{1}=0$. Then $z_{2} \geq 0$ and there exist a unique pair $\left(x, x^{*}\right)$ in $X$ such that

(i) $y_{1}=y_{2}=y_{3}=x$ and $y_{1}^{*}=y_{2}^{*}=y_{3}^{*}=x^{*}$;

(ii) $I$ is the segment $\left[x, x^{*}\right]$;

(iii) $\left[x, x^{*}\right]$ is the unique shortest segment joining $A_{p}$ to $A_{D}$.

(iv) Identifying $A_{i j}$ with $\mathbb{A}$ by a marked flat $f: \mathbb{A} \mapsto A_{i j}$ sending $\mathfrak{C}$ to $F_{j}$, in simple roots coordinates, we have $\overrightarrow{x x^{*}}=\left(-z_{2}, z_{2}\right)$. In particular $x^{*}$ is on the ray $\left[x, p_{i j}\right)$ from $x$ to $p_{i j}$.

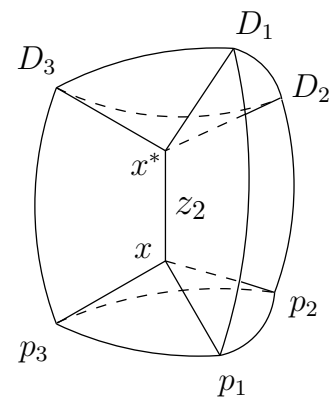

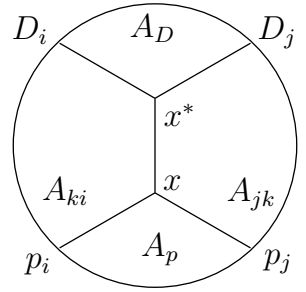

In the flat $A_{i j}$.

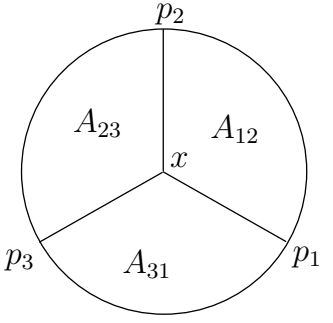

In the flat $A_{p}$.

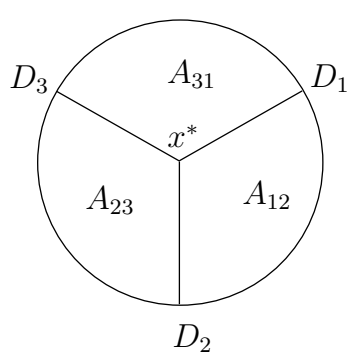

In the flat $A_{D}$.

FiguRE 1. Type "tripod"

Theorem 0.2 (Type "flat"). The intersection $A_{p} \cap A_{D}$ is nonempty if and only if $\left(z_{2}=0\right.$ or $\left.z_{3}=0\right)$, or, equivalently, if and only if $z_{2} \leq 0$. Then there exists a unique flat singular triangle $\Delta \subset X$ with vertices $x_{1}, x_{2}, x_{3}$ such that

(i) $A_{p} \cap A_{D}=\Delta$.

(ii) $A_{i j} \cap A_{i k}$ is the Weyl chamber from $x_{i}$ to $F_{i}$;

(iii) Let $i \in\{1,2,3\}$ and $j=i+1$. In a marked flat $f: \mathbb{A} \mapsto A_{i j}$ sending $\mathfrak{C}$ to $F_{j}$, in simple roots coordinates, we have $\overrightarrow{x_{i} x_{j}}=\left(z_{1}^{+}, z_{1}^{-}\right)$where $z_{1}^{+}=\max \left(z_{1}, 0\right)$ and $z_{1}^{-}=\max \left(-z_{1}, 0\right)$. In particular $x_{j}$ is on the ray from $x_{i}$ to $p_{j}$ (if $z_{1} \geq 0$ ) or $D_{j}$ (if $z_{1} \geq 0$ ).

(iv) The germs of Weyl chambers at $x_{i}$ respectively defined by $\Delta$ and $F_{i}$ are opposite (in the spherical building of directions at $x_{i}$ ). In particular there exists a flat containing $\Delta$, and containing $F_{i}$ in its boundary.

Furthermore if $z_{1} \geq 0$ we have $x_{i}=y_{i-1}=y_{i+1}^{*}$ for all $i$, and if $z_{1} \leq 0$ we have $x_{i}=y_{i+1}=y_{i-1}^{*}$ for all $i$. 
The intersections of each flat with the four other flats form a partition (i.e. a covering with disjoint interiors), which is described in Figure 1 for the type "tripod", and in Figure 2 for the type "flat" (see Proposition 4.2, Corollary 4.3 and Proposition 4.5).

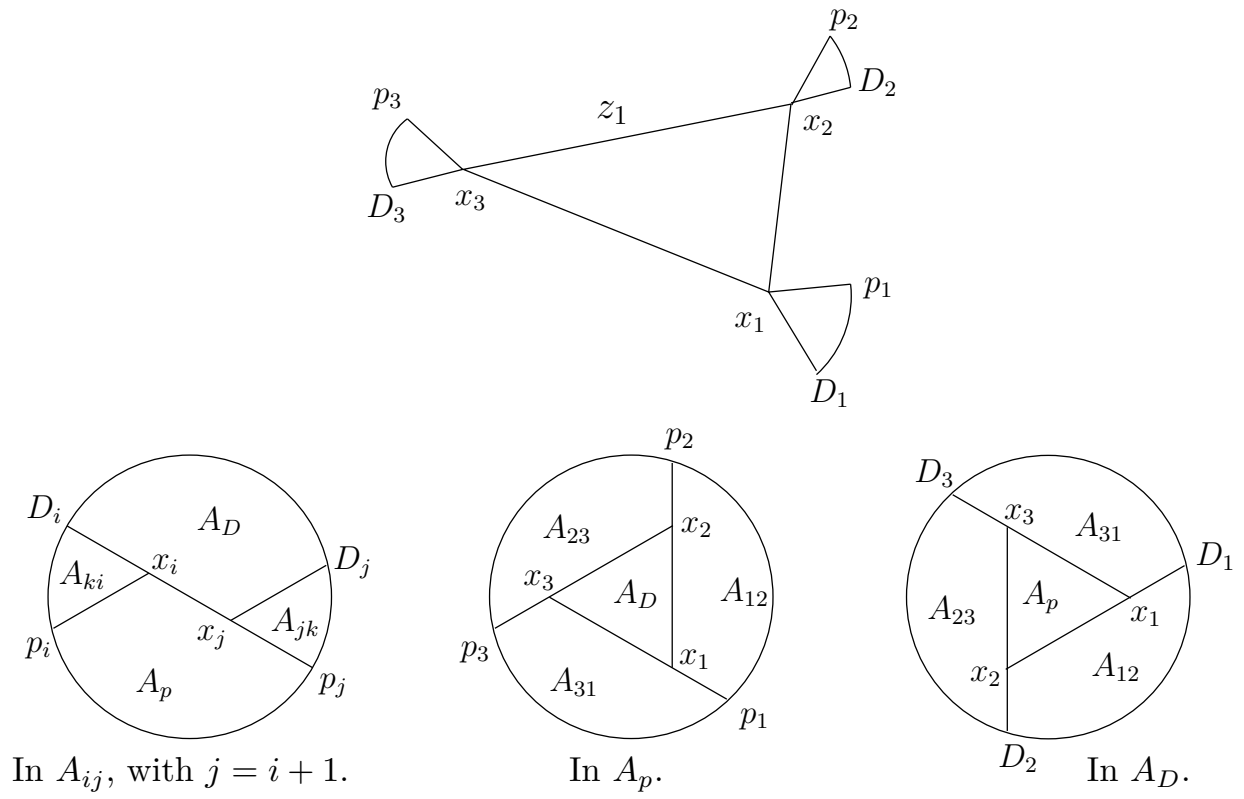

Figure 2. Type "flat", in the case where $z_{1} \geq 0$ (the case $z_{1} \leq 0$ is obtained from the case $z_{1} \geq 0$ by reversing the order of the flags $F_{i}$, i.e. by exchanging 1 and 3 and $i$ and $j$ in the above pictures).

The special case where the hypotheses of both Theorems 0.1 and 0.2 are satisfied corresponds to the case where $z_{1}=z_{2}=z_{3}=0$. Then the five flats intersect in a unique point $x$, and, in the spherical building of directions at $x$, the triple of chambers induced by $T=\left(F_{1}, F_{2}, F_{3}\right)$ is generic.

In particular we recover the characterization of [Bal08] for triples of points in $\partial_{\infty} X$ bounding a tripod in $X$. Note that M. Talbi established some analogous geometric classification for interior triangles in discrete Euclidean buildings of type $A_{2}$, see [Tal06].

Theorem 0.2 will be used in [Par15] to study actions of punctured surface groups on Euclidean buildings of type $A_{2}$. It allows us to give a metric interpretation, in the building, of Fock-Goncharov parameters associated with ideal triangulations. We are then able to construct in $X$ an invariant weakly convex cocompact 2-complex for large families of actions. Theorem 0.2 enables us to associate to each triangle of the triangulation a flat singular triangle in $X$, the complex is then obtained by connecting them gluing flat strips. This allows to describe length spectra for large families of degenerations of convex projective structures on surfaces.

We also show that generic quadruples of points in $\mathbb{P}$ (which will be called projective frames) define a nice center in $X$, with various characterizations, see Proposition 2.4 (this result generalizes to higer rank $\mathbb{R}$-buildings of type $A_{N-1}$ ). 
Aknowledgments. I would like to thank Frédéric Paulin for useful discussions and comments. I also want to thank the members of the Institut Fourier for their support.

\section{Preliminaries}

1A. The model flat $(\mathbb{A}, W)$ of type $A_{N-1}$. Let $N \geq 2$ be an integer. The model flat of type $A_{N-1}$ is the vector space $\mathbb{A}=\mathbb{R}^{N} / \mathbb{R}(1, \ldots, 1)$, endowed with the action of the Weyl group $W=\mathfrak{S}_{N}$ acting on $\mathbb{A}$ by permutation of coordinates (finite reflection group). We denote by $[\lambda]$ the projection in $\mathbb{A}$ of a vector $\lambda$ in $\mathbb{R}^{N}$. The vector space $\mathbb{A}$ may be identified with the hyperplane $\left\{\lambda=\left(\lambda_{1}, \ldots, \lambda_{N}\right) \in\right.$ $\left.\mathbb{R}^{N} / \sum_{i} \lambda_{i}=0\right\}$ of $\mathbb{R}^{N}$. Recall that a vector in $\mathbb{A}$ is called singular if it belongs to one the hyperplanes $\lambda_{i}=\lambda_{j}$, and regular otherwise. A (open) (vectorial) Weyl chamber of $\mathbb{A}$ is a connected component of regular vectors. We will call a sector a more general convex cone in $\mathbb{A}$, in particular the closed convex cone formed by the union of the closed Weyl chambers containing a given singular ray. The model Weyl chamber is the simplicial cone

$$
\mathfrak{C}=\left\{\lambda \in \mathbb{A} / \lambda_{1}>\cdots>\lambda_{N}\right\} .
$$

Its closure $\overline{\mathfrak{C}}$ is a strict fundamental domain for the action of $W$ on $\mathbb{A}$. Recall that two nonzero vectors $\lambda$ and $\lambda^{\prime}$ of $\mathbb{A}$ are called opposite if $\lambda^{\prime}=-\lambda$. Similarly, two Weyl chambers $C$ and $C^{\prime}$ of $\mathbb{A}$ are opposite if $C^{\prime}=-C$. The type of a vector $\lambda \in \mathbb{A}$ is its projection (modulo $W$ ) in $\overline{\mathfrak{C}}$.

We denote by $\partial \mathbb{A}$ the sphere of unitary vectors in $\mathbb{A}$, identified with the set $\mathbb{P}^{+}(\mathbb{A})=(\mathbb{A}-\{0\}) / \mathbb{R}_{>0}$ of rays issued from 0 , and by $\partial: \mathbb{A}-\{0\} \rightarrow \partial \mathbb{A}$ the corresponding projection. The type (of direction) of a nonzero vector $\lambda \in \mathbb{A}$ is its canonical projection in $\partial \overline{\mathfrak{C}}$.

We denote by $\left(\varepsilon_{1}, \ldots, \varepsilon_{N}\right)$ the canonical basis of $\mathbb{R}^{N}$. For $d=1, \ldots, N-1$, we will say that a nonzero vector in $\mathbb{A}$ (or a point in the sphere $\partial \mathbb{A}$ ) is singular of type $d$ if its canonical projection in $\partial \overline{\mathfrak{C}}$ is $\left[\varepsilon_{1}+\cdots+\varepsilon_{d}\right]$.

The simple roots (associated with $\mathfrak{C}$ ) are the following linear forms on $\mathbb{A}$

$$
\varphi_{i}: \lambda \mapsto \lambda_{i}-\lambda_{i+1}
$$

for $i=1, \ldots, N-1$. The set of simple roots is denoted by $\Lambda$. We will also use the $\operatorname{root} \varphi_{N}: \lambda \mapsto \lambda_{N}-\lambda_{1}$ satisfying

$$
\varphi_{1}+\cdots+\varphi_{N}=0 .
$$

The vector space $\mathbb{A}$ is endowed with the unique $W$-invariant Euclidean scalar product, which is well defined up to homothety (induced by the standard Euclidean scalar product of $\mathbb{R}^{N}$ ). We will normalize it by requiring that the simple roots have unit norm, i.e. the distance between the two hyperplanes with equation $\varphi_{i}=0$ and $\varphi_{i}=1$ is 1 for one (all) $i$. When $\operatorname{dim} \mathbb{A}=1$, we will identify $\mathbb{A}$ with $\mathbb{R}$ by the basis $\left\{\left[\varepsilon_{1}\right]\right\}$, i.e. by the map from $s \mapsto[(s, 0)]$ from $\mathbb{R}$ to $\mathbb{A}$, which is an isometry in the above normalization.

1B. Projective spaces. We here collect the notations and vocabulary for projective spaces, which will be used throughout this article. We refer to [Tit74, §6.2]. Let $\mathbb{P}$ be a projective space of dimension $N-1$, with $N \geq 2$. We denote by Flags $(\mathbb{P})$ 


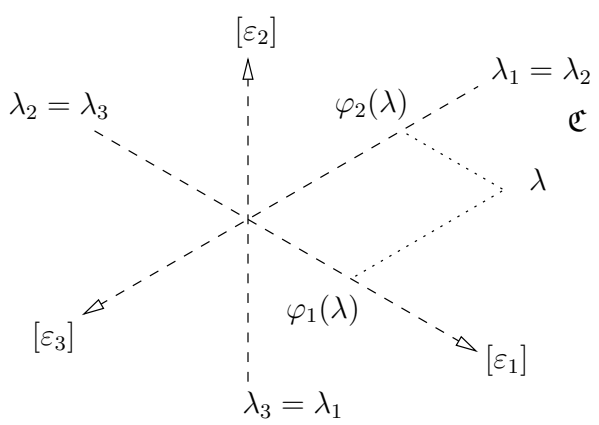

Figure 3 . The model flat $\mathbb{A}$ of type $A_{2}$ (for $N=3$ ), and simple roots coordinates. The arrows denote the singular directions of type 1 .

the set of flags of $\mathbb{P}$, that is increasing sequences $\left(V_{1}, \ldots, V_{M}\right)$ of proper linear subspaces of $\mathbb{P}$. We denote by $\mathbb{P}^{*}$ the dual projective space, whose set of points is the set of hyperplanes of $\mathbb{P}$.

Two maximal flags $\left(V_{1}, \ldots, V_{N-1}\right)$ and $\left(V_{1}^{\prime}, \ldots, V_{N-1}^{\prime}\right)$ are opposite if they are in generic position, that is if $V_{i} \oplus V_{n-i}^{\prime}=\mathbb{P}$ for all $i$. A finite subset $p_{1}, \ldots, p_{M}$ in $\mathbb{P}$, with $2 \leq M \leq N$, is called independent if it is not contained in any linear subspace of dimension $M-2$ of $\mathbb{P}$. Then it is contained in a unique $(M-1)$-dimensional linear subspace of $\mathbb{P}$, which will be denoted by $p_{1} \oplus \cdots \oplus p_{M}$. When $M=2$, we will also denote the line $p \oplus q$ by $p q$.

A frame of $\mathbb{P}$ is a independent $N$-tuple. A projective frame in $\mathbb{P}$ is a $(N+1)$-tuple $\left(p_{0}, p_{1}, \ldots, p_{N}\right)$ of points in $\mathbb{P}$ in generic position, i.e. such that the induced $N$-tuple $\left(p_{0}, \ldots, \widehat{p}_{i}, \ldots, p_{N}\right)$ is a frame in $\mathbb{P}$ for all $i$.

If $p$ is a point in $\mathbb{P}$, we denote by $\mathbb{P} / p$ the set of lines through $p$, which is a projective space of dimension $N-2$ whose linear subspaces are the linear subspaces of $\mathbb{P}$ containing $p$. The projection at $p$ is the corresponding projection $\operatorname{proj}_{p}: q \mapsto p q$ from $\mathbb{P}-\{p\}$ to $\mathbb{P} / p$. If $p$ is a point of $\mathbb{P}$ and $H \subset \mathbb{P}$ an hyperplane with $p \notin H$, then the projection $\operatorname{proj}_{p}$ induces a canonical isomorphism $\operatorname{proj}_{H p}: H \stackrel{\sim}{\rightarrow} \mathbb{P} / p$ (called perspectivity).

Note that if $\mathcal{F}=\left(p_{1}, \ldots, p_{M}\right)$ is independent in $\mathbb{P}$, then its projection $\operatorname{proj}_{p_{1}}(\mathcal{F})=$ $\left(p_{1} p_{2}, \ldots, p_{1} p_{M}\right)$ at $p_{1}$ is independent in $\mathbb{P} / p_{1}$. In particular the projection of a (projective) frame at one of its points is a (projective) frame.

1C. Spherical buildings of type $A_{N-1}$ and associated projective spaces. See $\left[\right.$ Tit74, §6]. A spherical building $\mathcal{B}$ of type $A_{N-1}$ is the building of flags of an associated projective space $\mathbb{P}=\mathbb{P}(\mathcal{B})$ of dimension $N-1$. For $d=0,1, \ldots, N-1$, the set of linear subspaces of dimension $d$ of $\mathbb{P}$ identifies with the subset of vertices of type $d+1$ of $\mathcal{B}$. In particular, the projective space $\mathbb{P}$ itself is identified with the set of vertices of type 1 of $\mathcal{B}$, and the dual projective space $\mathbb{P}^{*}$ is identified with the set of vertices of type $N-1$.

In the algebraic case, that is when $\mathcal{B}$ is the spherical building of flags of some vector space $V$ of dimension $N$ over a field $\mathbb{K}$, then $\mathbb{P}=\mathbb{P}(V)$.

A basic fact is that frames in $\mathbb{P}$ correspond to apartments of $\mathcal{B}$.

Recall that, in (the geometric realization modeled on $(\partial \mathbb{A}, W)$ of) a spherical building, any two points (resp. chambers) are contained in a common apartment, 
and that they are opposite if they are opposite in that apartment, that is, for two points $\xi$ and $\xi^{\prime}$, if and only if $\varangle\left(\xi, \xi^{\prime}\right)=\pi$ for the canonical metric $\varangle$ on $\mathcal{B}$. Note that $p \in \mathbb{P}$ and $H \in \mathbb{P}^{*}$ are opposite if and only if $\varangle(p, H)=\pi$, if and only if $p \notin H$. Two chambers are opposite if and only if they are opposite as maximal flags in $\mathbb{P}$. In particular, in the type $A_{2}$ case, two chambers $F_{1}=\left(p_{1}, D_{1}\right), F_{2}=\left(p_{2}, D_{2}\right)$ are opposite if and only if $p_{1} \notin D_{2}$ and $p_{2} \notin D_{1}$.

For any simplex $\sigma$ of $\mathcal{B}$ the residue $\operatorname{St}(\sigma)$ of $\sigma$ is the spherical building formed by the simplices of $\mathcal{B}$ containing $\sigma$. If $H$ is a hyperplane of $\mathbb{P}$, the residue $S t(H)$ of $H$ in $\mathcal{B}$ is the subset of flags of $\mathbb{P}$ containing $H$. It canonically identifies with the spherical building Flags $(H)$ of flags of $H$ by the map $\left(V_{1}, \ldots, V_{M}, H\right) \mapsto\left(V_{1}, \ldots, V_{M}\right)$. The residue $S t(p)$ of a point $p$ in $\mathbb{P}$ identifies canonically with the flag building Flags $(\mathbb{P} / p)$ of $\mathbb{P} / p$ by the map $\left(V_{1}=p, \ldots, V_{M}\right) \mapsto\left(V_{2} / p, \ldots, V_{M} / p\right)$. If $p \notin H$ then the projection $\operatorname{proj}_{p}$ induces a canonical isomorphism $\operatorname{proj}_{H p}: S t(H) \stackrel{\sim}{\rightarrow} S t(p)$ of spherical buildings (perspectivity).

1D. Euclidean buildings. Euclidean buildings considered in this article are (not necessarily discrete) Euclidean buildings of type $A_{N-1}$. We refer for example to [Par00] for the definition and properties of Euclidean buildings we use below (see also [Tit86], [KL97], [Rou09]). Recall that a Euclidean building of type $A_{N-1}$ is a $\operatorname{CAT}(0)$ metric space $X$ endowed with a (maximal) collection $\mathcal{A}$ of isometric embeddings $f: \mathbb{A} \rightarrow X$ called marked apartments, or marked flats by analogy with Riemannian symmetric spaces, satisfying the following properties

(A1) $\mathcal{A}$ is invariant by precomposition by $W_{a f f}$;

(A2) If $f$ and $f^{\prime}$ are two marked flats, then the transition map $f^{-1} \circ f^{\prime}$ is the restriction of an element of $W_{a f f}$;

(A3') Any two rays of $X$ are initially contained in a common marked flat;

where $W_{a f f}$ denotes the subgroup of all affine isomorphisms of $\mathbb{A}$ with linear part in $W$. The flats and the Weyl chambers) of $X$ are the images by the marked flats of $\mathbb{A}$ and $\mathfrak{C}$, respectively.

Algebraic case. Let $\mathbb{K}$ be an ultrametric field, i.e. a field endowed with an ultrametric absolute value $|\cdot|$ (not necessarily discrete). When $V$ is a finite $N$-dimensional vector space over $\mathbb{K}$, we denote by $X=X(V)$ the Euclidean building associated with $G=\operatorname{PGL}(V)$. We refer for example to [Par00] for the model of norms for $X$ (see [GI63], [BT84]). To each basis $\mathbf{v}$ of $V$ is then associated a marked flat $f_{\mathbf{v}}: \mathbb{A} \rightarrow A_{\mathbf{v}} \subset X$, such that, if $a$ is an element of $G$ with diagonal matrix $\operatorname{diag}\left(a_{1}, \ldots, a_{N}\right)$ in the basis $\mathbf{v}$, then $a$ translates the flat $A_{\mathbf{v}}$ by the vector

$$
\nu(a)=\left[\left(\log \left|a_{i}\right|\right)_{i}\right]
$$

in $\mathbb{A}$ (identifying the flat $A_{\mathbf{v}}$ with the model flat $\mathbb{A}$ through the marking $f_{\mathbf{v}}$ ).

From now to Section $1 H, X$ will denote a Euclidean building of type $A_{N-1}$.

1E. Spherical building and projective space at infinity. The CAT $(0)$ boundary $\partial_{\infty} X$ of $X$ is the geometric realization modeled on $(\partial \mathbb{A}, W)$ of a spherical building of type $A_{N-1}$ whose chambers are the boundaries of the Weyl chambers of $X$, and whose apartments are the boundaries of the flats of $X$. It will be identified with the building of flags on the associated projective space $\mathbb{P}=\mathbb{P}_{\infty}(X)$, whose points are the vertices of type 1 of $\partial_{\infty} X$. If $c_{+}$and $c_{-}$are opposite ideal chambers, then we denote by $A\left(c_{-}, c_{+}\right)$the unique flat joining $c_{-}$to $c_{+}$in $X$, that is, containing 
$c_{-}$and $c_{+}$in its boundary. If $\mathcal{F}$ is a frame of $\mathbb{P}$ or $\mathbb{P}^{*}$, then there is a unique flat $A(\mathcal{F})$ of $X$ containing $\mathcal{F}$ in its boundary.

1F. Local spherical building and projective space at a point. Recall that, in Euclidean buildings, two (unit speed) geodesic segments issued from a common point $x$ have zero angle if and only if they have same germ at $x$ (i.e. coincide in a neighborhood of $x$ ). A direction at $x \in X$ is a germ of nontrivial geodesic segment from $x$. A direction, geodesic segment, ray or line has a well-defined type (of direction) in $\partial \overline{\mathfrak{C}}$, which is its canonical projection (through a marked flat) in $\partial \overline{\mathfrak{C}}$. It is called singular or regular accordingly.

The space of directions at $x$ of $X$ is the quotient space of non trivial geodesic segments from $x$ for this relation, with the induced angular metric, and is denoted by $\Sigma_{x} X$. We denote by $\Sigma_{x}: X-\{x\} \rightarrow \Sigma_{x} X, y \rightarrow \Sigma_{x} y$, the associated projection. Its extension to the boundary at infinity will also be denoted by $\Sigma_{x}: \partial_{\infty} X \rightarrow \Sigma_{x} X$, $\xi \rightarrow \Sigma_{x} \xi$ and called the canonical projection.

The space of directions $\Sigma_{x} X$ inherits the structure of a spherical $A_{N-1}$-building, whose apartments are the germs $\Sigma_{x} A$ at $x$ of the flats $A$ of $X$ passing through $x$, and whose chambers are the germs $\Sigma_{x} C$ at $x$ of the Weyl chambers $C$ of $X$ with vertex $x$ (see for example [Par00]). The canonical projection $\Sigma_{x}: \partial_{\infty} X \rightarrow \Sigma_{x} X$ sends chambers to chambers (and, more generally, simplices to simplices) and preserves the type of points.

The local projective space $\mathbb{P}_{x}=\mathbb{P}_{x}(X)$ at $x$ is the projective space of dimension $N-1$ associated with the spherical building $\Sigma_{x} X$ of type $A_{N-1}$ (see $\S 1 \mathrm{C}$ ). Its underlying set is the set of vertices of type 1 of $\Sigma_{x} X$.

The canonical projection $\Sigma_{x}: \partial_{\infty} X \rightarrow \Sigma_{x} X$ induces (by restriction to vertices) a surjective morphism (of projective spaces) $\Sigma_{x}: \mathbb{P} \rightarrow \mathbb{P}_{x}$ from the projective space at infinity $\mathbb{P}$ to the local projective space $\mathbb{P}_{x}$ at $x$. Note that, in particular, if $\mathcal{F}$ is a frame of $\mathbb{P}$, then $x$ belongs to the associated flat $A(\mathcal{F})$ if and only if $\Sigma_{x}(\mathcal{F})$ is a frame of $\mathbb{P}_{x}$.

1G. Transverse spaces at infinity. See for example [Tit86, §8], [Lee00, 1.2.3], [MSv14, §4]. Let $\xi$ be a vertex of $\partial_{\infty} X$ of type 1 or $N-1$, i.e. either a point $p$ in the projective plane at infinity $\mathbb{P}$ or a hyperplane $H$ of $\mathbb{P}$.

The transverse space $X_{\xi}$ at $\xi$ may be defined, from the metric viewpoint (as in $[$ Lee00, 1.2.3]), as the quotient space of the set of all rays to $\xi$ by the pseudodistance $d_{\xi}$ given by

$$
d_{\xi}\left(r_{1}, r_{2}\right)=\inf _{t_{1}, t_{2}} d\left(r_{1}\left(t_{1}\right), r_{2}\left(t_{2}\right)\right) .
$$

We denote by $\pi_{\xi}: X \rightarrow X_{\xi}$ the canonical projection (which maps $x$ to the class of the unique ray from $x$ to $\xi$ ). The space $X_{\xi}$ is a Euclidean building of type $A_{N-2}$, whose flats are the projections to $X_{\xi}$ of the flats of $X$ containing a ray to $\xi$. In particular, when $X$ is of type $A_{2}$, the transverse space $X_{\xi}$ is an $\mathbb{R}$-tree, and we will call it the transverse tree at $\xi$.

In the algebraic case, i.e. when $X=X(V)$, the transverse space $X_{H}$ canonically identifies with the building $X(H)$ of $H$, where $H$ is seen as an hyperplane of $V$, and $X_{p}$ identifies with $X(V / p)$, where $p$ is seen as a 1-dimensional subspace of $V$.

The spherical building $\partial_{\infty} X_{\xi}$ at infinity of $X_{\xi}$ identifies canonically with the residue $S t(\xi)$ of $\xi$. In particular, if $p$ is a point in $\mathbb{P}$, the projective space at infinity of $X_{p}$ identifies with $\mathbb{P} / p$, and if $H$ is an hyperplane of $\mathbb{P}$, the projective space at infinity of $X_{H}$ identifies with $H$. 
If $\mathcal{F}=\left(p_{1}, \ldots, p_{N}\right)$ is a frame in $\mathbb{P} \subset \partial_{\infty} X$, then the projection on $X_{p_{1}}$ of the flat $A\left(p_{1}, \ldots, p_{N}\right)$ is the flat defined by the projection $\operatorname{proj}_{p_{1}}(\mathcal{F})=\left(p_{1} p_{2}, \ldots, p_{1} p_{N}\right)$ of the frame $\mathcal{F}$, i.e. $\pi_{p_{1}}(A(\mathcal{F}))=A\left(\operatorname{proj}_{p_{1}}(\mathcal{F})\right)$.

We now describe the canonical isomorphism $\pi_{\xi^{-} \xi^{+}}: X_{\xi^{-}} \stackrel{\sim}{\rightarrow} X_{\xi^{+}}$for opposite points $\xi^{-}$and $\xi^{+}$of $\partial_{\infty} X$. The union $F_{\xi^{-} \xi^{+}}$of all geodesics joining $\xi^{-}$to $\xi^{-}$is a convex closed subspace and a subbuilding, whose flats are the flats of $X$ containing a geodesic joining $\xi^{-}$to $\xi^{-}$(see [KL97, prop. 4.8.1] and [Par12, 2.2.1]). We denote by $F_{\xi^{-} \xi^{+}}=X^{\xi^{-} \xi^{+}} \times \mathbb{R}$ the canonical decomposition (see [Par11, 1.2.10]). The restriction of the projection $\pi_{\xi^{+}}$to $F_{\xi^{-} \xi^{+}}$is surjective and factorizes through the projection on the first factor, inducing a canonical isomorphism of Euclidean buildings $X^{\xi^{-} \xi^{+}} \stackrel{\sim}{\rightarrow} X_{\xi^{+}}$. We similarly have a isomorphism $X^{\xi^{-} \xi^{+}} \stackrel{\sim}{\rightarrow} X_{\xi^{-}}$, so it induces a canonical isomorphism $\pi_{\xi^{-} \xi^{+}}: X_{\xi^{-}} \stackrel{\sim}{\rightarrow} X_{\xi^{+}}$. It is easy to see that the map $\pi_{\xi^{-} \xi^{+}}$extends to the boundaries at infinity of $X_{\xi^{-}}$and $X_{\xi^{+}}$by the canonical isomorphism of spherical buildings $\operatorname{proj}_{\xi^{-} \xi^{+}}: S t\left(\xi^{-}\right) \stackrel{\sim}{\rightarrow} S t\left(\xi^{+}\right)$(perspectivity).

1H. The $\mathbb{A}$-valued Busemann cocycle. Let $c$ be a chamber at infinity of $X$. We now define the $\mathbb{A}$-valued Busemann cocycle

$$
B_{c}: X \times X \rightarrow \mathbb{A}
$$

associated to $c$. It can be simply defined from canonical retractions as

$$
B_{c}(x, y):=r(y)-r(x)
$$

where $r: X \rightarrow \mathbb{A}$ is any canonical retraction centered at $c$, sending $c$ to $\partial \mathfrak{C}$ (see [Par00, Prop. 1.19])). More precisely, the Buseman cocycle at $c$ is characterized by the property:

$$
B_{c}\left(f(\lambda), f^{\prime}\left(\lambda^{\prime}\right)\right)=\lambda^{\prime}-\lambda
$$

for any two marked flats $f, f^{\prime}: \mathbb{A} \rightarrow X$ sending $\partial \mathfrak{C}$ to $c$ and such that $f=f^{\prime}$ on some subchamber of $\mathfrak{C}$.

We clearly have

$$
B_{c}(x, z)=B_{c}(x, y)+B_{c}(y, z) .
$$

When $\operatorname{dim} \mathbb{A}=1$, it coincides with the usual Busemann cocycle, which is defined for $\xi \in \partial_{\infty} X$ by

$$
B_{\xi}(x, y)=\lim _{z \rightarrow \xi} d(x, z)-d(y, z) .
$$

In the type $A_{2}$ case, the simple root coordinates of $\mathbb{A}$-valued Busemann cocycles may be determined by projecting in transverse trees at infinity, using the following relations (using the normalization of the metric).

$$
\begin{aligned}
& \varphi_{1}\left(B_{(p, D)}(x, y)\right)=B_{p}\left(\pi_{D}(x), \pi_{D}(y)\right) \\
& \varphi_{2}\left(B_{(p, D)}(x, y)\right)=B_{D}\left(\pi_{p}(x), \pi_{p}(y)\right) .
\end{aligned}
$$

We now turn to cross ratios. 
1I. Cross ratio on the boundary of a tree. See [Tit86, §7], and in a more general setting [Ota92], [Bou96]. In this section, we suppose that $X$ is an $\mathbb{R}$-tree. Given three distinct ideal points $\xi_{1}, \xi_{2}, \xi_{3}$ in $\partial_{\infty} X$, we denote by $c\left(\xi_{1}, \xi_{2}, \xi_{3}\right)$ the center of the ideal triple $\left(\xi_{1}, \xi_{2}, \xi_{3}\right)$, that is the unique common intersection point of the three geodesic lines joining two of the three points. Note that $c\left(\xi_{1}, \xi_{2}, \xi_{3}\right)$ is the (orthogonal) projection of $\xi_{3}$ on the geodesic joining $\xi_{1}$ to $\xi_{2}$. We denote by $B_{\xi}(x, y)$ the Busemann cocycle (see $\left.\S 1 \mathrm{H}\right)$.

Define the cross ratio of four pairwise distinct points $\xi_{1}, \xi_{2}, \xi_{3}, \xi_{4}$ in $\partial_{\infty} X$ by

$$
\boldsymbol{\beta}\left(\xi_{1}, \xi_{2}, \xi_{3}, \xi_{4}\right)=\frac{1}{2}\left(\ell_{12}-\ell_{23}+\ell_{34}-\ell_{41}\right)
$$

where $\ell_{i j}$ is the length of the geodesic in $X$ from $\xi_{i}$ to $\xi_{j}$ after removing disjoint fixed horoballs centered at each $\xi_{k}$. It does not dependend on the choice of the horoballs since the horoballs centered at a given point are equidistant along the rays to that point.

The cross ratio naturally extends to nondegenerate quadruples, that are quadruples $\left(\xi_{1}, \xi_{2}, \xi_{3}, \xi_{4}\right)$ without triple point (i.e. any three of the points are not equal), which is equivalent to the following condition:

$$
\left(\xi_{1} \neq \xi_{4} \text { and } \xi_{2} \neq \xi_{3}\right) \text { or }\left(\xi_{1} \neq \xi_{2} \text { and } \xi_{3} \neq \xi_{4}\right) \text {. }
$$

We then set

$$
\boldsymbol{\beta}\left(\xi_{1}, \xi_{2}, \xi_{3}, \xi_{4}\right)=\left\{\begin{array}{cl}
0 & \text { when } \xi_{1}=\xi_{3} \text { or } \xi_{2}=\xi_{4} \\
-\infty & \text { when } \xi_{1}=\xi_{2} \text { or } \xi_{3}=\xi_{4} \\
+\infty & \text { when } \xi_{1}=\xi_{4} \text { or } \xi_{2}=\xi_{3}
\end{array} .\right.
$$

We now recall some basic properties that we will use.

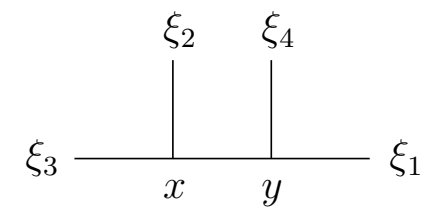

The cocycle identity is

$$
\boldsymbol{\beta}\left(\xi_{1}, \xi_{2}, \xi_{3}, \xi_{4}\right)+\boldsymbol{\beta}\left(\xi_{1}, \xi_{4}, \xi_{3}, \xi_{5}\right)=\boldsymbol{\beta}\left(\xi_{1}, \xi_{2}, \xi_{3}, \xi_{5}\right) .
$$

The cross ratio $\beta$ is left unchanged by the double transpositions and changed to $-\boldsymbol{\beta}$ by the transpositions (13) and (24). We now consider the behaviour under cyclic permutations of the three last terms. We have

$$
\boldsymbol{\beta}\left(\xi_{1}, \xi_{2}, \xi_{3}, \xi_{4}\right)+\boldsymbol{\beta}\left(\xi_{1}, \xi_{4}, \xi_{2}, \xi_{3}\right)+\boldsymbol{\beta}\left(\xi_{1}, \xi_{3}, \xi_{4}, \xi_{2}\right)=0 .
$$

Moreover, the following ultrametricity property (specific to the case of trees) is easy to prove using (1.3) (see [Tit86, §7, prop. 3]):

$$
\begin{aligned}
& \text { If } \boldsymbol{\beta}\left(\xi_{1}, \xi_{2}, \xi_{3}, \xi_{4}\right)>0 \text {, then } \boldsymbol{\beta}\left(\xi_{1}, \xi_{3}, \xi_{4}, \xi_{2}\right)=0 \\
& \text { and } \boldsymbol{\beta}\left(\xi_{1}, \xi_{4}, \xi_{2}, \xi_{3}\right)=-\boldsymbol{\beta}\left(\xi_{1}, \xi_{2}, \xi_{3}, \xi_{4}\right)
\end{aligned}
$$

Note that (1.5) is equivalent (under (1.4)) to

$$
\boldsymbol{\beta}\left(\xi_{1}, \xi_{2}, \xi_{3}, \xi_{4}\right) \leq \max \left(0,-\boldsymbol{\beta}\left(\xi_{1}, \xi_{4}, \xi_{2}, \xi_{3}\right)\right) \text {. }
$$


which in the algebraic case follows from the symmetry properties of the cross ratio under 3-cyclic permutations (1.9).

1J. Algebraic case: link with usual cross ratio. Suppose that $X$ is the tree $X(V)$ associated with a 2-dimensional vector space $V$ over an ultrametric field $\mathbb{K}$ (see Section 1D). Then $\partial_{\infty} X$ identifies with the projective line $\mathbb{P}(V)$.

The usual cross ratio $\mathbf{b}$ on $\mathbb{P}(V)$ of a nondegenerate quadruple of points (see (1.2)) is defined by (following the convention of [FG07], and taking values in $\mathbb{K} \cup\{\infty\}$ )

$$
\mathbf{b}\left(a_{1}, a_{2}, a_{3}, a_{4}\right)=\frac{\left(a_{1}-a_{2}\right)\left(a_{3}-a_{4}\right)}{\left(a_{1}-a_{4}\right)\left(a_{2}-a_{3}\right)}
$$

in any affine chart $\mathbb{P}(V) \stackrel{\sim}{\rightarrow} \mathbb{K} \cup\{\infty\}$, so that $\mathbf{b}(\infty,-1,0, a)=a$.

The cross ratio $\boldsymbol{\beta}$ defined in Section 1I will then be called the geometric cross ratio, to distinguish it from $\mathbf{b}$, which will be called the algebraic cross ratio. They are then related as follows:

$$
\boldsymbol{\beta}\left(\xi_{1}, \xi_{2}, \xi_{3}, \xi_{4}\right)=\log \left|\mathbf{b}\left(\xi_{1}, \xi_{2}, \xi_{3}, \xi_{4}\right)\right| .
$$

Proof. Let $x_{4}=c\left(\xi_{3}, \xi_{1}, \xi_{2}\right)$ and $x_{2}=c\left(\xi_{3}, \xi_{1}, \xi_{4}\right)$. In a suitable basis $\mathbf{v}=\left(v_{1}, v_{2}\right)$ of $V$, we have in homogeneous coordinates $\xi_{1}=[1: 0], \xi_{3}=[0: 1], \xi_{2}=[-1: 1]$ and $\xi_{4}=[b: 1]$, where $b=\mathbf{b}\left(\xi_{1}, \xi_{2}, \xi_{3}, \xi_{4}\right)$. Then $g=\left(\begin{array}{cc}-b & 0 \\ 0 & 1\end{array}\right)$ fixes $\xi_{1}$ and $\xi_{3}$ and sends $\xi_{2}$ to $\xi_{4}$. Hence $g\left(x_{4}\right)=x_{2}$. In the flat $A\left(\xi_{3}, \xi_{1}\right)$ identified with $\mathbb{A}=\mathbb{R}^{2} / \mathbb{R}(1,1)$ by the marked flat $f_{\mathbf{v}}$, we have $\overrightarrow{x_{4} x_{2}}=\nu(g)=[(\log |b|, 0)]$, hence $\overrightarrow{x_{4} x_{2}}=\log |b|$ as needed.

We recall that the algebraic cross ratio $\mathbf{b}$ satisfies the following symmetry properties: It is left unchanged by the double transpositions and changed to $\mathbf{b}^{-1}$ by the transpositions (13) and (24). Furthermore we have an additional symmetry under 3 -cycles not satisfied by the geometric cross ratio:

$$
\begin{aligned}
& \mathbf{b}\left(a_{1}, a_{3}, a_{4}, a_{2}\right)=-1-\mathbf{b}\left(a_{1}, a_{2}, a_{3}, a_{4}\right)^{-1} \\
& \mathbf{b}\left(a_{1}, a_{4}, a_{2}, a_{3}\right)=-\left(1+\mathbf{b}\left(a_{1}, a_{2}, a_{3}, a_{4}\right)\right)^{-1} .
\end{aligned}
$$

1K. Cross ratio on the boundary of an $A_{2}$-Euclidean building. See [Tit86]. Let $X$ be a Euclidean building of type $A_{2}$, and $\mathbb{P}$ the associated projective plane at infinity.

Let $\left(p_{1}, p_{2}, p_{3}, p_{4}\right)$ be a nondegenerate quadruple of points of $\mathbb{P}$ on a common line $D$. Then their cross ratio $\boldsymbol{\beta}\left(p_{1}, p_{2}, p_{3}, p_{4}\right)$ (i.e. projective valuation in [Tit86]) is by definition their cross ratio as ideal points of the transverse tree $X_{D}$. The cross ratio of a nondegenerate quadruple of lines in $\mathbb{P}$ passing through a common point $p$ is similarly defined as their cross ratio as ideal points of the transverse tree $X_{p}$.

The main additional property is that perspectivities preserve cross ratio, which follows from the fact that perspectivities extend isometries between the transverse trees (see Section 1G):

Proposition 1.1. Let $p$ be a point of $\mathbb{P}$ and $D$ a line of $\mathbb{P}$ with $p \notin D$. The canonical isomorphisms (perspectivities) $\operatorname{proj}_{p D}: S t(D) \stackrel{\sim}{\rightarrow} S t(p), q \mapsto p q$ and $\operatorname{proj}_{D p}: S t(p) \stackrel{\sim}{\rightarrow} S t(D), L \mapsto D \cap L$, preserve the cross ratio $\boldsymbol{\beta}$, i.e.

$$
\begin{gathered}
\boldsymbol{\beta}\left(p_{1}, p_{2}, p_{3}, p_{4}\right)=\boldsymbol{\beta}\left(p p_{1}, p p_{2}, p p_{3}, p p_{4}\right) \\
\boldsymbol{\beta}\left(D_{1}, D_{2}, D_{3}, D_{4}\right)=\boldsymbol{\beta}\left(D \cap D_{1}, D \cap D_{2}, D \cap D_{3}, D \cap D_{4}\right)
\end{gathered}
$$




\section{Some BASIC IDEAL CONFIGURATIONS}

2A. Extension of orthogonal projection to the boundary in $\operatorname{CAT}(0)$ spaces. In this section $X$ is a general CAT(0) metric space, and we prove the following basic property: the usual orthogonal projection onto a proper convex subset $Y \subset X$ extends to the boundary outside the closed $\frac{\pi}{2}$-neighborhood of $\partial_{\infty} Y$ for the Tits metric (note that the projection is no longer unique). This property is quite elementary but we did not see it in the classical litterature, so we include the proof. We refer to the book [BH99] for CAT(0) spaces.

We denote by $\partial_{\infty} X$ the $\operatorname{CAT}(0)$ boundary of $X$, and by $\varangle_{T i t s}(\xi, \eta)$ the Tits angle between two ideal points $\xi, \eta \in \partial_{\infty} X$. For a subset $A$ of $\partial_{\infty} X$, we define $\varangle_{T i t s}(\xi, A)=\inf _{\eta \in A} \varangle_{T i t s}(\xi, \eta)$.

Definition 2.1. Let $Y$ be a subspace of $X$ and $\xi \in \partial_{\infty} X$ an ideal point. We say that a point $x \in Y$ is an orthogonal projection of $\xi$ on $Y$ if $\varangle_{x}(\xi, y) \geq \frac{\pi}{2}$ for all $y \in Y-\{x\}$.

Proposition 2.2. Let $Y$ be a convex subspace of a $C A T(0)$ space $X$ which is proper for the induced metric, and $\xi$ in $\partial_{\infty} X$. Suppose that $\varangle_{T i t s}\left(\xi, \partial_{\infty} Y\right)>\frac{\pi}{2}$. Then there exists an orthogonal projection $x$ of $\xi$ on $Y$.

Proof. Consider a sequence $\left(x_{n}\right)$ converging to $\xi$ in $X$, and let $y_{n}$ be the orthogonal projection of $x_{n}$ on $Y$. If $\left(y_{n}\right)_{n \in \mathbb{N}}$ is not bounded then, up to passing to a subsequence, $y_{n}$ converges to $\eta$ in $\partial_{\infty} Y$. Then for any fixed $y$ in $Y$ we have $\varangle_{y}\left(\xi, y_{n}\right) \leq \frac{\pi}{2}$ for all $n$, hence $\varangle_{y}(\xi, \eta) \leq \frac{\pi}{2}$. Therefore $\varangle_{\text {Tits }}(\xi, \eta) \leq \frac{\pi}{2}$. Thus $\left(y_{n}\right)_{n \in \mathbb{N}}$ is bounded, hence, since $Y$ is proper, it has a converging subsequence, and the limit point $x$ is then an orthogonal projection of $\xi$ on $Y$.

2B. Centers of generic $(N+1)$-tuples. In this section, we show that the notion of center of ideal triples in trees extends to Euclidean buildings of type $A_{N-1}$, for generic $(N+1)$-tuples of points (or hyperplanes) in the associated projective space at infinity (Proposition 2.4).

Let $X$ be a Euclidean building of type $A_{N-1}$, and $\mathbb{P}$ be its projective space at infinity (i.e., the set of singular points of type 1 in $\partial_{\infty} X$, see Section 1). Recall from Section 1B that a projective frame in a projective space of dimension $N-1$ is a generic $(N+1)$-tuple of points.

We first observe that the orthogonal projection of a point of $\mathbb{P}$ on a flat of $X$ exists under a simple necessary and sufficient condition.

Proposition 2.3. Let $A$ be a flat of $X$ and $p \in \mathbb{P}$. Let $\left(p_{1}, \ldots, p_{N}\right)=\left(\partial_{\infty} A\right) \cap \mathbb{P}$ be the points of type 1 in $\partial_{\infty} A$. Then $p$ admits an orthogonal projection on $A$ if and only if $\left(p, p_{1}, \ldots, p_{N}\right)$ is a projective frame.

The analoguous property is also valid for points $H \in \mathbb{P}^{*}$. Note that these properties also hold in symmetric spaces of type $A_{N-1}$.

Proof. If $p \in H$ for some hyperplane $H$ in $\mathbb{P}^{*} \cap \partial_{\infty} A$, then $p$ and $H$ are in a common chamber of the spherical building $\partial_{\infty} X$, and, as the diameter $d$ of the model spherical Weyl chamber $\partial \overline{\mathfrak{C}}$ is stricly less that $\pi / 2$ (for the angle metric), we have $\varangle_{\text {Tits }}(p, H)<\pi / 2$, hence the orthogonal projection does not exist. Else, for every hyperplane $H$ in $\mathbb{P}^{*} \cap \partial_{\infty} A$, we have $p \notin H$, hence $\varangle_{T i t s}(p, H)=\pi$, which implies that since $\varangle_{T i t s}(p, \eta) \geq \pi-d>\pi / 2$ for all $\eta \in \partial_{\infty} A$, and the orthogonal projection exists by Proposition 2.2. 
We now turn to the main result of this section.

Proposition 2.4. Let $\mathcal{F}=\left(p_{0}, p_{1}, \ldots, p_{N}\right)$ be a projective frame in $\mathbb{P} \subset \partial_{\infty} X$. For each $i \in\{0, \ldots, N\}$ let $A_{i}$ be the unique flat of $X$ through $\left(p_{0}, \ldots, \widehat{p}_{i}, \ldots, p_{N}\right)$. There exists a unique point $x \in X$ satisfying the following equivalent conditions.

(i) $x \in \cap_{i} A_{i}$;

(ii) For all $i$ and for all $H$ in $\partial_{\infty} A_{i} \cap \mathbb{P}^{*}$ the angle $\varangle_{x}\left(p_{i}, H\right)$ is $\pi$;

(iii) The $(N+1)$-tuple $\Sigma_{x} \mathcal{F}=\left(\Sigma_{x} p_{i}\right)_{i=0, \ldots, N}$ of directions at $x$ form a projective frame in $\mathbb{P}_{x}$;

(iv) For all $i$, the point $x$ is an orthogonal projection of $p_{i}$ on the flat $A_{i}$;

(v) There exists $i$ such that $x$ is an orthogonal projection of $p_{i}$ on $A_{i}$.

We will call $x$ the center of the projective frame $\mathcal{F}=\left(p_{0}, p_{1}, \ldots, p_{N}\right)$ and denote it by $c\left(p_{0}, p_{1}, \ldots, p_{N}\right)$ or $c(\mathcal{F})$.

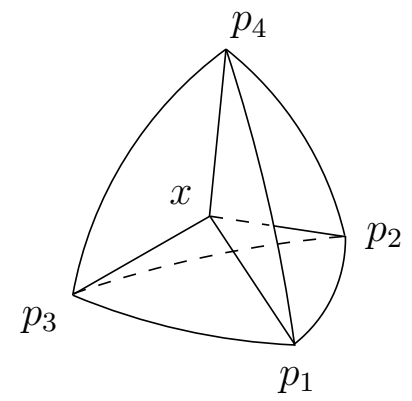

Figure 4 . The center $x \in X$ of a projective frame $\left(p_{1}, p_{2}, p_{3}, p_{4}\right)$ (for $N=3$ ).

Proof. The existence of $x$, as an orthogonal projection of $p_{0}$ on $A_{0}$, is ensured by Proposition 2.3.

For $i \neq j$, denote by $H_{i j}$ the hyperplane $\oplus_{k \neq i, j} p_{k}$ in the projective space $\mathbb{P}$. Let $x \in X$. Conditions (iii) and (i) are equivalent (see Section $1 \mathrm{~F}$ ).

We first show (i) $\Rightarrow$ (ii): Fix $i$ and $H \in \mathbb{P}^{*}$ in $\partial_{\infty} A_{i}$. The opposite of $H$ in $\partial_{\infty} A_{i}$ is some $p_{j}$. Then $H=H_{i j}$, so $H$ is also the opposite of $p_{i}$ in the apartment $\partial_{\infty} A_{j}$. As $x \in A_{j}$, we then have $\varangle_{x}\left(p_{i}, H\right)=\pi$. We now prove (ii) $\Rightarrow$ (iii): First recall that for $p \in \mathbb{P}$ and $H \in \mathbb{P}^{*}$, we have $\varangle_{x}\left(p_{i}, H\right)=\pi$ if and only if $\Sigma_{x} p \notin \Sigma_{x} H$ in the projective space $\mathbb{P}_{x}$. So (ii) means that $\Sigma_{x} p_{i} \notin \Sigma_{x} H_{i j}$ for all $i \neq j$. Let $U_{i}$ be the minimal linear subspace of the projective space $\mathbb{P}_{x}$ containing $\Sigma_{x} p_{0}, \ldots, \Sigma_{x} p_{i}$. Then, for $i \leq N-1$, we have that $\Sigma_{x} p_{i}$ is not in $U_{i-1}$, else $\Sigma_{x} p_{i}$ would belong to $\Sigma_{x} H_{i, i+1}$. Hence $\left(\Sigma_{x} p_{0}, \ldots, \Sigma_{x} p_{i}\right)$ is independent in $\mathbb{P}_{x}$ by induction on $i$. Therefore $\left(\Sigma_{x} p_{0}, \ldots, \Sigma_{x} p_{N-1}\right)$ is a frame, and (iii) follows by permuting the $p_{i}$.

We now prove (ii) $\Rightarrow$ (iv). Let $i \in\{0, \ldots, N\}$. Let $v \in \Sigma_{x} A_{i}$. Let $C \subset A_{i}$ be a closed Weyl chamber with vertex $x$ containing $v$. Let $H \in \mathbb{P}^{*}$ be the singular point of type $N-1$ in $\partial_{\infty} C$. Then $\varangle_{x}\left(p_{i}, H\right)=\pi$, hence $\varangle_{x}\left(p_{i}, v\right) \geq \pi-d>\frac{\pi}{2}$, as the diameter $d$ of $\partial \overline{\mathfrak{C}}$ is stricly less that $\pi / 2$.

(iv) $\Rightarrow(\mathrm{v})$ is clear. Assume now that (v) holds. For $j \neq i$ in $\{0, \ldots, N\}$, as $\varangle_{x}\left(p_{i}, H_{i j}\right) \geq \frac{\pi}{2}$, the direction $\Sigma_{x} p_{i}$ is not in a closed chamber of $\Sigma_{x} X$ containing $\Sigma_{x} H_{i j}$. Hence by type considerations we must have $\varangle_{x}\left(p_{i}, H_{i j}\right)=\pi$. So (ii) holds. 
So the equivalence of all assertions is proven. We now prove the uniqueness of $x$. Suppose that $x^{\prime}$ is another point of $X$ with the same properties, and $x^{\prime} \neq x$. We proved above that we have then $\varangle_{x}\left(p_{i}, x^{\prime}\right)>\frac{\pi}{2}$ and $\varangle_{x^{\prime}}\left(p_{i}, x\right)>\frac{\pi}{2}$, which is impossible.

We now state some properties of centers of projective frames. Consider a projective frame $\mathcal{F}=\left(p_{0}, p_{1}, \ldots, p_{N}\right)$ in $\mathbb{P}$, and let $x \in X$ be its center. Let $A_{i}=$ $A\left(p_{0}, \ldots, \widehat{p}_{i}, \ldots, p_{N}\right)$ be the $N+1$ associated flats in $X$. We first describe the intersections of the flats $A_{i}$ with $A_{0}$.

Proposition 2.5. For $i=1 \ldots N$, let $S_{i}$ be the sector with base-point $x$ on $\left\{p_{1}, \ldots, \widehat{p}_{i}, \ldots, p_{N}\right\}$, i.e. the convex hull of the rays from $x$ to these points. And let $H_{i}=p_{1} \oplus \cdots \oplus \widehat{p}_{i} \oplus \cdots \oplus p_{N}$ denote the point in $\partial_{\infty} A_{0}$ opposite to $p_{i}$. For $i \in\{1, \ldots, N\}$, we have:

(i) Let $y$ be an interior point of $S_{i}$. Then $\Sigma_{y} p_{0}=\Sigma_{y} p_{i}$.

(ii) For $y \in A_{0}$, we have $y \in A_{0} \cap A_{i}$ if and only if $\Sigma_{y} p_{0}$ is opposite to $\Sigma_{y} H_{i}$;

(iii) $A_{0} \cap A_{i}=S_{i}$;

In particular, the intersections $A_{0} \cap A_{i}, i=1 \ldots N$, form a partition (i.e. a covering with disjoint interiors) of $A_{i}$.

Note that the sector $S_{i}$ is the union of the Weyl chambers of the flat $A_{0}$ based at $x$ and containing the singular ray to $H_{i}$.

Proof. The inclusion $S_{i} \subset A_{0} \cap A_{i}$ is clear since $x \in A_{0} \cap A_{i}$ and $p_{j}$ is in $\partial_{\infty} A_{0} \cap \partial_{\infty} A_{i}$ for $j \neq i$ in $\{1, \ldots, N\}$.

If $y$ is an interior point of $S_{i}$, then in the local spherical building $\Sigma_{y} X$ at $y$, we have that $\Sigma_{y} p_{0} \in \Sigma_{y} A_{0}$. Moreover, $y \in A_{i}$ as previously observed, so $\Sigma_{y} p_{0}$ is opposite to $\Sigma_{y} H_{i}$ (in $\Sigma_{y} A_{i}$ ). Hence $\Sigma_{y} p_{0}$ is equal to the opposite of $\Sigma_{y} H_{i}$ in $\Sigma_{y} A_{0}$, which is $\Sigma_{y} p_{i}$, proving (i).

We now prove (ii): In $\mathbb{P}_{y}$, the points $\left(\Sigma_{y} p_{1}, \ldots, \Sigma_{y} p_{N}\right)$ form a frame (since $\left.y \in A_{0}\right)$. Hence the $N-1$ points $\left(\Sigma_{y} p_{1}, \ldots, \widehat{\Sigma_{y} p_{i}}, \ldots, \Sigma_{y} p_{N}\right)$ are independent Therefore $\left(\Sigma_{y} p_{0}, \ldots, \widehat{\Sigma_{y} p_{i}}, \ldots, \Sigma_{y} p_{N}\right)$ is a frame in $\mathbb{P}_{y}$ (i.e. $\left.y \in A_{i}\right)$ if and only if $\Sigma_{y} p_{0} \notin \Sigma_{y} H_{i}$.

We finish by proving the remaining inclusion $A_{0} \cap A_{i} \subset S_{i}$ : The $S_{i}$ clearly form a partition of $A_{0}$. So it is enough to prove that that $A_{0} \cap A_{i}$ does not meet the interior of $S_{j}$ for $j \neq i$. Else, at such a point $y$, by (i), we would have $\Sigma_{y} p_{0}=\Sigma_{y} p_{j}$, which is not opposite to $\Sigma_{y} H_{i}$, providing a contradiction.

The following proposition shows that the notion of center of projective frames behaves well with respect to projections to transverse spaces at infinity.

Proposition 2.6. For each $i$, the projection of $x$ in the transverse building at infinity $X_{p_{i}}$ is the center of the projective frame of $\partial_{\infty} X_{p_{i}}$ formed by the projections $\operatorname{proj}_{p_{i}}\left(p_{j}\right)=p_{i} p_{j}$ of the $p_{j}, j \neq i$, that is:

$$
\pi_{p_{i}}\left(c\left(p_{0}, p_{1}, \ldots, p_{N}\right)\right)=c\left(p_{i} p_{0}, p_{i} p_{1}, \ldots, \widehat{p_{i} p_{i}}, \ldots, p_{i} p_{N}\right) .
$$

Proof. For all $j \neq i$, the ray from $x$ to $p_{i}$ is in the flat $A_{j}$ hence its projection $\pi_{p_{i}}(x)$ in the transverse building $X_{p_{i}}$ is in $\pi_{p_{i}}\left(A_{j}\right)$, which is the flat defined by the frame $\operatorname{proj}_{p_{i}}\left(p_{k}\right)=p_{i} p_{k}, k \neq i, j$. 
In the algebraic case, i.e. when $X$ is the Euclidean buiding $X(V)$ associated with some vector space $V$ of dimension $N$ over an ultrametric field $\mathbb{K}$, we have the following characterization of the center as a norm on $V$.

Proposition 2.7. Let $\mathcal{F}=\left(p_{0}, p_{1}, \ldots, p_{N}\right)$ be a projective frame in $\mathbb{P}=\mathbb{P}(V)$. The center of $\mathcal{F}$ is the norm $\eta$ on $V$ canonically associated to any basis $\mathbf{v}=\left(v_{i}\right)_{i=1, \ldots N}$ of $V$ such that $p_{i}=\left[v_{i}\right]$ for $1 \leq i \leq N$ and $p_{0}=\left[v_{1}+\cdots+v_{N}\right]$ in $\mathbb{P}(V)$, i.e. the norm defined by

$$
\eta\left(\sum_{i=1}^{N} a_{i} v_{i}\right)=\max _{1 \leq i \leq N}\left|a_{i}\right| .
$$

Proof. Let $\mathbf{v}=\left(v_{1}, \ldots, v_{N}\right)$ be a basis of $V$ such that $p_{i}=\left[v_{i}\right]$ and $p_{0}=\left[v_{1}+\right.$ $\left.\cdots+v_{N}\right]$ in $\mathbb{P}(V)$. Let $\eta$ be the associated canonical norm on $V$. We clearly have $\eta \in A_{0}$ by the definition of marked flats in the model of norms. Let $g$ be the element of $\operatorname{GL}(V)$ sending the basis $\mathbf{v}$ to the basis $\left(v_{1}, \ldots, v_{N-1}, v_{1}+\cdots+v_{N}\right)$. Then $g$ preserves the norm $\eta$ and sends $A_{0}$ to $A_{N}$ and hence $\eta$ is in the flat $A_{N}$. Permuting the basis $v$, we similarly get that $\eta$ is in the flat $A_{i}$ for all $i \neq 0$.

Remark 2.8. By duality, the similar properties hold for generic $(N+1)$-tuples (projective frames) in $\mathbb{P}^{*} \subset \partial_{\infty} X$.

2C. Projecting two ideal points onto a flat. From now on we return to the case where $N=3$ (type $A_{2}$ ).

Proposition 2.9. Let $\left(p_{1}, p_{2}, p_{3}\right)$ be a independent triple in $\mathbb{P}$. Let $p, q$ be two points in $\mathbb{P}$, in generic position relatively to the $p_{i}$ (i.e. not on any of the lines $\left.p_{i} p_{j}\right)$. Denote by $x$ and $y$ the respective orthogonal projections of $p$ and $q$ on the flat $A=A\left(p_{1}, p_{2}, p_{3}\right)$. Identify $A$ with $\mathbb{A}$ by a marked flat sending $\partial \mathfrak{C}$ to $\left(p_{1}, p_{1} p_{2}\right)$. Then the roots coordinates of $\overrightarrow{x y}$ are given by the three natural cross ratios at the vertices of the triangle:

$$
\begin{gathered}
\varphi_{1}(\overrightarrow{x y})=\boldsymbol{\beta}\left(p_{3} p_{1}, p_{3} p, p_{3} p_{2}, p_{3} q\right), \\
\varphi_{2}(\overrightarrow{x y})=\boldsymbol{\beta}\left(p_{1} p_{2}, p_{1} p, p_{1} p_{3}, p_{1} q\right), \\
\varphi_{3}(\overrightarrow{x y})=\boldsymbol{\beta}\left(p_{2} p_{3}, p_{2} p, p_{2} p_{1}, p_{2} q\right) .
\end{gathered}
$$

The analogous dual result holds for projections of two lines of $\mathbb{P}$ on a flat (exchanging the roles of points and lines in $\mathbb{P}$ ).

Proof. Projecting on the transverse tree $X_{p_{1}}$ in direction $p_{1}$, we have

$$
\varphi_{2}(\overrightarrow{x y})=\varphi_{2}\left(B_{\left(p_{1}, p_{1} p_{2}\right)}(x, y)\right)=B_{p_{1} p_{2}}\left(\pi_{p_{1}}(x), \pi_{p_{1}}(y)\right)
$$

by (1.1). Since the projections of $x$ and $y$ on the tree $X_{p_{1}}$ are the respective centers of the ideal triples $\left(p_{1} p_{2}, p_{1} p_{3}, p_{1} p\right)$ and $\left(p_{1} p_{2}, p_{1} p_{3}, p_{1} q\right)$ (Proposition 2.6), we have

$$
B_{p_{1} p_{2}}\left(\pi_{p_{1}}(x), \pi_{p_{1}}(y)\right)=\boldsymbol{\beta}\left(p_{1} p_{2}, p_{1} p, p_{1} p_{3}, p_{1} q\right)
$$

by $(1.3)$, hence $\varphi_{2}(\overrightarrow{x y})=\boldsymbol{\beta}\left(p_{1} p_{2}, p_{1} p, p_{1} p_{3}, p_{1} q\right)$. The remaining assertions follow by applying cyclic permutation, since

$$
\begin{gathered}
\varphi_{1}\left(B_{\left(p_{1}, p_{1} p_{2}\right)}(x, y)\right)=\varphi_{2}\left(B_{\left(p_{3}, p_{3} p_{1}\right)}(x, y)\right) \\
\varphi_{3}\left(B_{\left(p_{1}, p_{1} p_{2}\right)}(x, y)\right)=\varphi_{2}\left(B_{\left(p_{2}, p_{2} p_{3}\right)}(x, y)\right) .
\end{gathered}
$$

For the projections of a point and a line, we have the following result. 
Proposition 2.10. Let $F_{-}=\left(p_{-}, D_{-}\right)$and $F_{+}=\left(p_{+}, D_{+}\right)$be two oppposite flags in $\mathbb{P}$ and $A$ the flat in $X$ joining them, identified with $\mathbb{A}$ by a marked flat sending $\partial \mathfrak{C}$ to $F_{+}$. Let $p$ be a point and $D$ a line in $\mathbb{P}$ in generic position with respect to $F_{-}$ and $F_{+}$, (i.e. $p$ does not belong to any of the lines $p_{-} p_{+}, D_{-}, D_{+}$, and $D$ does not contain any of the points $\left.D_{-} \cap D_{+}, p_{-}, p_{+}\right)$.

Denote by $x$ and $x^{*}$ the respective orthogonal projections of $p$ and $D$ on $A$. Then in simple roots coordinates we have

$$
\begin{gathered}
\overrightarrow{x x^{*}}=\left(z_{-}, z_{+}\right), \\
\text {with } z_{-}=\boldsymbol{\beta}\left(p_{+}, D_{+} \cap\left(p_{-} p\right), D_{+} \cap D_{-}, D_{+} \cap D\right) \\
=\boldsymbol{\beta}\left(D_{-}, p_{-} \oplus\left(D_{+} \cap D\right), p_{-} p_{+}, p_{-} p\right) \\
\text { and } z_{+}=\boldsymbol{\beta}\left(p_{-}, D_{-} \cap D, D_{-} \cap D_{+}, D_{-} \cap\left(p_{+} p\right)\right) \\
=\boldsymbol{\beta}\left(D_{+}, p_{+} p, p_{+} p_{-}, p_{+} \oplus\left(D_{-} \cap D\right)\right) .
\end{gathered}
$$

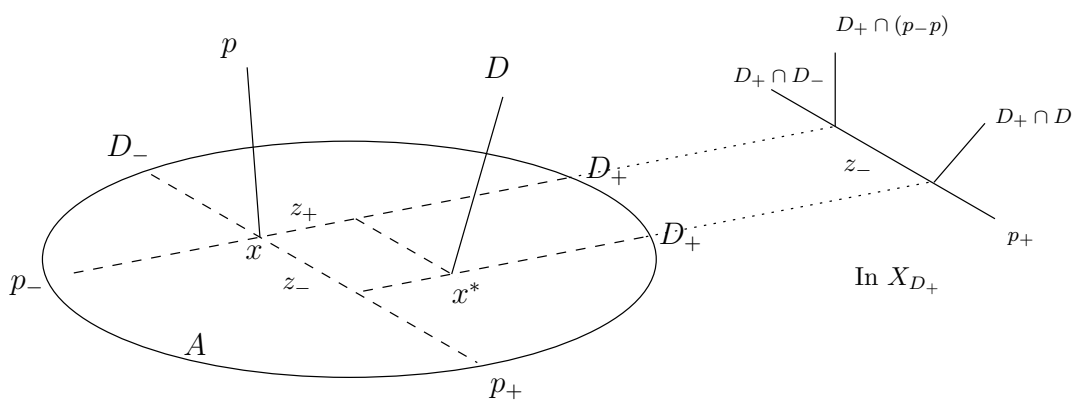

In $X$

Figure 5. Projecting a point and a line on a flat.

Proof. See Figure 5. The projection of $x$ on the transverse tree $X_{p_{-}}$is the center of the ideal triple $\left(p_{-} p_{+}, p_{-}\left(D_{-} \cap D_{+}\right), p_{-} p\right)$, and the projection of $x^{*}$ on the tree $X_{D_{+}}$is the center of the ideal triple $\left(p_{+}, D_{+} \cap D_{-}, D_{+} \cap D\right)$ (Proposition 2.6). As $x$ lies on a geodesic from $p_{-}$to $D_{+}$, we have

$$
\begin{aligned}
\pi_{D_{+}}(x) & =\pi_{D_{+}, p_{-}}\left(\pi_{p_{-}}(x)\right) \\
& =\pi_{D_{+}, p_{-}}\left(c\left(p_{-} p_{+}, p_{-}\left(D_{-} \cap D_{+}\right), p_{-} p\right)\right) \\
& =c\left(p_{+}, D_{-} \cap D_{+}, D_{+} \cap\left(p_{-} p\right)\right) .
\end{aligned}
$$

Then projecting on the transverse tree $X_{D_{+}}$we have

$$
\varphi_{1}\left(\overrightarrow{x x^{*}}\right)=B_{p_{+}}\left(\pi_{D_{+}}(x), \pi_{D_{+}}\left(x^{*}\right)\right)=\boldsymbol{\beta}\left(p_{+}, D_{+} \cap\left(p_{-} p\right), D_{+} \cap D_{-}, D_{+} \cap D\right)
$$

as needed. The remaining assertions have identical proofs.

\section{TRiple RATIO OF A triple of ideAl Chambers}

In this section, we introduce the (geometric) triple ratio of a nondegenerate triple of ideal chambers in a a real Euclidean building $X$ of type $A_{2}$, establish its basic properties, and the links with the usual $\mathbb{K}$-valued (algebraic) triple ratio of triples of flags (see e.g. [FG07]) in the algebraic case $\mathbb{P}=\mathbb{P}\left(\mathbb{K}^{3}\right)$. 
We first give a precise definition of nondegenerate and generic triples of flags in an arbitrary projective plane $\mathbb{P}$.

3A. Nondegenerate and generic triples of flags. Let $\mathbb{P}$ be a projective plane and $T=\left(F_{1}, F_{2}, F_{3}\right)$ be a triple of flags $F_{i}=\left(p_{i}, D_{i}\right)$ in $\mathbb{P}$. We will denote by $p_{i j}$ the point $D_{i} \cap D_{j}$ (resp. $D_{i j}$ the line $p_{i} p_{j}$ ), when defined.

The natural nondegeneracy condition on the triple $\left(F_{1}, F_{2}, F_{3}\right)$ for the triple ratios to be well defined is the following:

$$
\text { (ND) either } \forall i, p_{i} \notin D_{i+1} \text { or } \forall i, p_{i} \notin D_{i-1} \text {. }
$$

This condition is clearly equivalent to: the points are pairwise distinct, the lines are pairwise distinct, none of the points is on the three lines (i.e. $D_{i} \cap D_{j} \neq p_{k}$ for all $\{i, j, k\}=\{1,2,3\})$ and none of the lines contains the three points (i.e. $p_{i} p_{j} \neq D_{k}$ for all $i, j, k)$. We will then say that the triple $\left(F_{1}, F_{2}, F_{3}\right)$ is nondegenerate.

It is easy to check that the triple $T$ defines then a nondegenerate quadruple $\left(D_{i}, p_{i} p_{j}, p_{i} p_{j k}, p_{i} p_{k}\right)$ of lines through each point $p_{i}$, and a nondegenerate quadruple $\left(p_{i}, D_{i} \cap D_{j}, D_{i} \cap D_{j k}, D_{i} \cap D_{k}\right)$ of points on each line $D_{i}$.

The triple of flags $T=\left(F_{1}, F_{2}, F_{3}\right)$ is generic if the flags $F_{i}=\left(p_{i}, D_{i}\right)$ are pairwise opposite, the points $\left(p_{i}\right)_{i}$ are not collinear and the lines $\left(D_{i}\right)_{i}$ are not concurrent. In particular, $T$ is then nondegenerate, and the induced quadruples of points on each line (resp. of lines through each point) are generic (i.e. pairwise distinct).

3B. Algebraic triple ratio. When $\mathbb{P}=\mathbb{P}\left(\mathbb{K}^{3}\right)$ is the projective plane associated with an arbitrary field $\mathbb{K}$, the algebraic triple ratio of a nondegenerate triple of flags $T=\left(F_{1}, F_{2}, F_{3}\right)$ (see Section 3A), with values in $\mathbb{K} \cup\{\infty\}$, is defined by (see [FG06, $\S 9.4])$

$$
\operatorname{Tri}\left(F_{1}, F_{2}, F_{3}\right)=\frac{\tilde{D}_{1}\left(\tilde{p}_{2}\right) \tilde{D}_{2}\left(\tilde{p}_{3}\right) \tilde{D}_{3}\left(\tilde{p}_{1}\right)}{\tilde{D}_{1}\left(\tilde{p}_{3}\right) \tilde{D}_{2}\left(\tilde{p}_{1}\right) \tilde{D}_{3}\left(\tilde{p}_{2}\right)}
$$

where $\tilde{p}_{i}$ is any vector in $\mathbb{K}^{3}$ representing $p_{i}$ and $\tilde{D}_{i}$ is any linear form in $\left(\mathbb{K}^{3}\right)^{*}$ representing $D_{i}$, and $F_{i}=\left(p_{i}, D_{i}\right)$. It is invariant under cyclic permutation of the flags and inversed by reversing the order

$$
\operatorname{Tri}\left(F_{3}, F_{2}, F_{1}\right)=\operatorname{Tri}\left(F_{1}, F_{2}, F_{3}\right)^{-1} .
$$

It may be expressed as the following cross ratio

$$
\operatorname{Tri}\left(F_{1}, F_{2}, F_{3}\right)=\mathbf{b}\left(D_{1}, p_{1} p_{2}, p_{1} p_{23}, p_{1} p_{3}\right) .
$$

$3 \mathrm{C}$. Geometric triple ratio. We suppose now that the projective plane $\mathbb{P}$ is the projective plane at infinity of some a real Euclidean building $X$ of type $A_{2}$, possibly exotic. Let $\beta$ be the associated geometric cross ratio on $\mathbb{P}$ (see Section $1 \mathrm{~K})$. Let $T=\left(F_{1}, F_{2}, F_{3}\right)$ be a nondegenerate triple of ideal chambers of $X$, i.e. a nondegenerate triple of flags $F_{i}=\left(p_{i}, D_{i}\right)$ in $\mathbb{P}$.

The idea is to define the geometric triple ratio of $T$ by analogy with the expression of the algebraic triple ratio as a cross ratio (3.1), replacing $\mathbf{b}$ by $\boldsymbol{\beta}$, in such a way that, in the algebraic case, the geometric triple ratio of a triple $T$ with algebraic triple ratio $Z$ should be $\log |Z|$. But for the purpose of geometric classification, this geometric cross ratio $\boldsymbol{\beta}\left(D_{1}, p_{1} p_{2}, p_{1} p_{23}, p_{1} p_{3}\right)$ alone will not retain enough information. In particular, in contrast to the algebraic cross ratio, it does not determine the geometric cross ratios obtained from the original 4-tuple by cyclic permutations of the three last arguments, which in the algebraic case are $\log \left|1+Z^{-1}\right|$ and 
$-\log |1+Z|$, see (1.9), and have geometric significance. For example, in the algebraic case, it will not distinguish between two triples $T$ and $T^{\prime}$ with respective algebraic triple ratios $Z=-1$ and $Z^{\prime}=-1+a$ with $|a|<1$.

In order to retain this information we define the geometric triple ratio of $T$ as the triple

$$
\operatorname{tri}(T)=\left(\operatorname{tri}_{m}(T)\right)_{m=1,2,3}
$$

where

$$
\begin{aligned}
& \operatorname{tri}_{1}\left(F_{1}, F_{2}, F_{3}\right)=\boldsymbol{\beta}\left(D_{1}, p_{1} p_{2}, p_{1} p_{23}, p_{1} p_{3}\right) \\
& \operatorname{tri}_{2}\left(F_{1}, F_{2}, F_{3}\right)=\boldsymbol{\beta}\left(D_{1}, p_{1} p_{3}, p_{1} p_{2}, p_{1} p_{23}\right) \\
& \operatorname{tri}_{3}\left(F_{1}, F_{2}, F_{3}\right)=\boldsymbol{\beta}\left(D_{1}, p_{1} p_{23}, p_{1} p_{3}, p_{1} p_{2}\right)
\end{aligned}
$$

are the geometric cross ratios obtained from $\left(D_{1}, p_{1} p_{2}, p_{1} p_{23}, p_{1} p_{3}\right)$ by cyclic permutation of the three last lines. Note these cross ratios are well defined, since the four lines $D_{1}, p_{1} p_{2}, p_{1} p_{23}, p_{1} p_{3}$ are well defined and form a nondegenerate quadruple of lines through $p_{1}$ (see Section $3 \mathrm{~A}$ above).

The following proposition gathers the properties of the geometric triple ratio, and show in particular that this invariant is in fact 1-dimensional, as it takes values in one of the three rays $\mathbb{R}_{+}(0,1,-1), \mathbb{R}_{+}(-1,0,1)$, and $\mathbb{R}_{+}(1,-1,0)$.

Proposition 3.1. The following hold.

(i) The geometric triple ratio is invariant by cyclic permutations of the flags, i.e. for $m=1,2,3$,

$$
\operatorname{tri}_{m}\left(F_{2}, F_{3}, F_{1}\right)=\operatorname{tri}_{m}\left(F_{1}, F_{2}, F_{3}\right) ;
$$

(ii) Exchanging two flags, we have

$$
\begin{aligned}
& \operatorname{tri}_{1}\left(F_{1}, F_{3}, F_{2}\right)=-\operatorname{tri}_{1}\left(F_{1}, F_{2}, F_{3}\right), \\
& \operatorname{tri}_{2}\left(F_{1}, F_{3}, F_{2}\right)=-\operatorname{tri}_{3}\left(F_{1}, F_{2}, F_{3}\right)
\end{aligned} ;
$$

(iii) We have $\operatorname{tri}_{1}(T)+\operatorname{tri}_{2}(T)+\operatorname{tri}_{3}(T)=0$;

(iv) For all $m \in \mathbb{Z} / 3 \mathbb{Z}$, if $\operatorname{tri}_{m}(T)>0$, then we have $\operatorname{tri}_{m-1}(T)=0$ and $\operatorname{tri}_{m+1}(T)=-\operatorname{tri}_{m}(T)<0$.

In order to prove this proposition, in particular, the invariance of the triple ratio by cyclic permutation of the flags, we first introduce the natural dual invariants given by the cross ratios of the natural induced quadruple of points on the line $D_{1}$ (that is, exchanging the role of points and lines):

$$
\begin{aligned}
& \operatorname{tri}_{1}^{*}\left(F_{1}, F_{2}, F_{3}\right)=\boldsymbol{\beta}\left(p_{1}, D_{2} \cap D_{1}, D_{23} \cap D_{1}, D_{3} \cap D_{1}\right) \\
& \operatorname{tri}_{2}^{*}\left(F_{1}, F_{2}, F_{3}\right)=\boldsymbol{\beta}\left(p_{1}, D_{3} \cap D_{1}, D_{2} \cap D_{1}, D_{23} \cap D_{1}\right) \\
& \operatorname{tri}_{3}^{*}\left(F_{1}, F_{2}, F_{3}\right)=\boldsymbol{\beta}\left(p_{1}, D_{23} \cap D_{1}, D_{3} \cap D_{1}, D_{2} \cap D_{1}\right) .
\end{aligned}
$$

The following property is straigthforward.

$$
\begin{aligned}
\operatorname{tri}_{1}^{*}\left(F_{1}, F_{3}, F_{2}\right) & =-\operatorname{tri}_{1}^{*}\left(F_{1}, F_{2}, F_{3}\right) \\
\operatorname{tri}_{2}^{*}\left(F_{1}, F_{3}, F_{2}\right) & =-\operatorname{tri}_{3}^{*}\left(F_{1}, F_{2}, F_{3}\right) .
\end{aligned}
$$

We will need the following property showing that the invariants behave nicely under duality.

Lemma 3.2. For $m=1,2,3$, we have $\operatorname{tri}_{m}^{*}\left(F_{1}, F_{2}, F_{3}\right)=\operatorname{tri}_{m}\left(F_{3}, F_{2}, F_{1}\right)$. 
Proof of Lemma 3.2. By invariance under perspectivities and double transpositions, we have

$$
\begin{aligned}
\operatorname{tri}_{1}^{*}\left(F_{1}, F_{2}, F_{3}\right) & =\boldsymbol{\beta}\left(p_{1}, D_{2} \cap D_{1}, D_{23} \cap D_{1}, D_{3} \cap D_{1}\right) \\
& =\boldsymbol{\beta}\left(p_{1} p_{3}, p_{12} p_{3}, D_{23}, D_{3}\right) \\
& =\boldsymbol{\beta}\left(D_{3}, p_{2} p_{3}, p_{12} p_{3}, p_{1} p_{3}\right) \\
& =\operatorname{tri}_{1}\left(F_{3}, F_{2}, F_{1}\right) .
\end{aligned}
$$

The proof of $\operatorname{tri}_{m}^{*}\left(F_{1}, F_{2}, F_{3}\right)=\operatorname{tri}_{m}\left(F_{3}, F_{2}, F_{1}\right)$ for $m=2,3$ is similar.

We now turn to the proof of Proposition 3.1.

Proof of Proposition 3.1. Assertions (iii) and (iv) follow immediately from the properties of the cross ratio $\beta$ under cyclic permutation of the three last points (see (1.4) and (1.5)).

Assertion (ii) follows immediately from the definition and from the symmetries of the cross ratio.

We finally prove (i) of Proposition 3.1. Using (ii), Lemma 3.2 and (3.2), we have

$$
\begin{aligned}
\operatorname{tri}_{1}\left(F_{2}, F_{3}, F_{1}\right) & =-\operatorname{tri}_{1}\left(F_{2}, F_{1}, F_{3}\right) \\
& =-\operatorname{tri}_{1}^{*}\left(F_{3}, F_{1}, F_{2}\right) \\
& =\operatorname{tri}_{1}^{*}\left(F_{3}, F_{2}, F_{1}\right)=\operatorname{tri}_{1}\left(F_{1}, F_{2}, F_{3}\right), \\
\operatorname{tri}_{2}\left(F_{2}, F_{3}, F_{1}\right) & =-\operatorname{tri}_{3}\left(F_{2}, F_{1}, F_{3}\right) \\
& =-\operatorname{tri}_{3}^{*}\left(F_{3}, F_{1}, F_{2}\right) \\
& =\operatorname{tri}_{2}^{*}\left(F_{3}, F_{2}, F_{1}\right)=\operatorname{tri}_{2}\left(F_{1}, F_{2}, F_{3}\right) .
\end{aligned}
$$

The case where $m=3$ is similar to the case $m=2$.

3D. Geometric triple ratio from algebraic triple ratio. When $\mathbb{P}$ is the projective plane on some field $\mathbb{K}$ endowed with some ultrametric absolute value, and $\boldsymbol{\beta}=\log |\mathbf{b}|$ where $\mathbf{b}$ is the usual $\mathbb{K}$-valued cross ratio on $\mathbb{P}$, the three geometric triple ratios $\operatorname{tri}_{m}(T), m=1,2,3$ of $T$ are obtained from the single algebraic triple ratio $Z=\operatorname{Tri}(T)$ of $T$ by the relations

$$
\begin{aligned}
& \operatorname{tri}_{1}(T)=\log |Z| \\
& \operatorname{tri}_{2}(T)=\log \left|\frac{1}{1+Z}\right|=-\log |1+Z| \\
& \operatorname{tri}_{3}(T)=\log \left|1+Z^{-1}\right|,
\end{aligned}
$$

which are easily derived from the expression of algebraic triple ratio as a cross ratio (3.1) and from the symmetry properties of the algebraic cross ratio (1.9).

Remark 3.3. Note that the geometric invariants do not determine the triple of flags up to automorphisms of $\mathbb{P}$ (unlike the usual (algebraic) triple ratio): for example in the algebraic case $\mathbb{P}=\mathbb{P}\left(\mathbb{K}^{3}\right)$, take $T$ with triple ratio $Z \in \mathbb{K}$ with $|Z|>1$ and $T^{\prime}$ with triple ratio $Z^{\prime}=Z a$ where $a \in \mathbb{K}$ with $|a|=1$ and $a \neq 1$. Then $T$ and $T^{\prime}$ are not in the same $\mathrm{PGL}\left(\mathbb{K}^{3}\right)$-orbit, but have the same three geometric invariants, as $\operatorname{tri}_{1}(T)=\log |Z|=\operatorname{tri}_{1}\left(T^{\prime}\right), \operatorname{tri}_{2}(T)=-\log |Z|=\operatorname{tri}_{2}\left(T^{\prime}\right), \operatorname{tri}_{3}(T)=0=\operatorname{tri}_{3}\left(T^{\prime}\right)$. 


\section{Proof of the main Result}

In this section we prove Theorems 0.1 and 0.2 . Let $X$ be a Euclidean building of type $A_{2}$ and $T=\left(F_{1}, F_{2}, F_{3}\right)$ be a generic triple of flags in the projective plane $\mathbb{P}$ at infinity of $X$. We denote by $z_{m}=\operatorname{tri}_{m}\left(F_{1}, F_{2}, F_{3}\right), m=1,2,3$, its geometric triple ratio, and by $A_{i j}=A\left(F_{i}, F_{j}\right), A_{p}=A\left(p_{1}, p_{2}, p_{3}\right)$ and $A_{D}=A\left(D_{1}, D_{2}, D_{3}\right)$ the five associated flats.

We first define the six associated points in $X$.

4A. Associated points in the building. For $\{i, j, k\}=\{1,2,3\}$, denote by $y_{k}$ the center in $X$ of the projective frame $\left(p_{1}, p_{2}, p_{3}, p_{i j}\right)$, where $p_{i j}=D_{i} \cap D_{j}$, and by $y_{k}^{*}$ the center of the projective frame $\left(D_{1}, D_{2}, D_{3}, D_{i j}\right)$, where $D_{i j}=p_{i} p_{j}$, as defined in Proposition 2.4. In particular the point $y_{k}$ is the orthogonal projection of $p_{i j}$ on $A_{p}$, the point $y_{k}^{*}$ is the orthogonal projection of $D_{i j}$ on $A_{D}$, the point $y_{k}$ is the orthogonal projection of $p_{k}$ on $A_{i j}=A\left(p_{i}, p_{j}, p_{i j}\right)$, and the point $y_{k}^{*}$ is the orthogonal projection of $D_{k}$ on $A_{i j}=A\left(D_{i}, D_{j}, D_{i j}\right)$.

4B. In the flat $A_{i j}$. We now link the respective position of the points $y_{k}$ and $y_{k}^{*}$ in the flat $A_{i j}$ to the geometric triple ratio of $T$. Suppose that the indices $i, j, k$ respect the cyclic order, i.e. that $(i, j, k)=(123)$ as cyclic permutations. We identify $A_{i j}$ with the model flat $\mathbb{A}$ by a marked flat $f_{i j}: \mathbb{A} \rightarrow A_{i j}$ sending $\partial \mathfrak{C}$ to $F_{j}$. For $x, y$ in $A_{i j} \simeq \mathbb{A}$, we define then $\overrightarrow{x y}=y-x=B_{F_{j}}(x, y)$. Recall that $\left(\varepsilon_{1}, \varepsilon_{2}, \varepsilon_{3}\right)$ denotes the canonical basis of $\mathbb{R}^{3}$. In particular, the directions of $p_{i}, p_{i j}$ and $p_{j}$ are respectively identified with the directions of $\left[\varepsilon_{1}\right]$, $\left[\varepsilon_{2}\right]$, and $\left[\varepsilon_{3}\right]$ in $\mathbb{A}$.

Proposition 4.1. The following holds.

(i) In simple roots coordinates, we have $\overrightarrow{y_{k}^{*} y_{k}}=\left(z_{2}, z_{3}\right)$;

(ii) For $m=1,2,3$, if $z_{m}>0$ then $\overrightarrow{y_{k} y_{k}^{*}}=z_{m}\left[\varepsilon_{m}\right]$. In particular $y_{k}^{*}$ is on one of the three singular rays of type 1 issued from $y_{k}$ (i.e the rays to $p_{i}, p_{j}$ and $p_{i j}$ ).

Proof. As $y_{k}$ and $y_{k}^{*}$ are the respective orthogonal projections on the flat $A_{i j}$ of $p_{k}$ and $D_{k}$, by Proposition 2.10 and cyclic invariance of the geometric triple ratio, we have

$$
\begin{aligned}
\varphi_{1}\left(\overrightarrow{y_{k}^{*} y_{k}}\right) & =\boldsymbol{\beta}\left(D_{i}, p_{i} p_{k}, p_{i} p_{j}, p_{i} p_{j k}\right)=\operatorname{tri}_{2}\left(F_{i}, F_{j}, F_{k}\right)=z_{2} \\
\text { and } \varphi_{2}\left(\overrightarrow{y_{k}^{*} y_{k}}\right) & =\boldsymbol{\beta}\left(D_{j}, p_{j} p_{k i}, p_{j} p_{i}, p_{j} p_{k}\right)=\operatorname{tri}_{3}\left(F_{j}, F_{k}, F_{i}\right)=z_{3} .
\end{aligned}
$$

Assertion (ii) follows, since we have then $z_{m-1}=0$ and $z_{m+1}=-z_{m}$ by ultrametricity of the geometric triple ratio (Proposition 3.1(iv)).

We now describe the intersections of $A_{i j}$ with the four other flats (see Figures 1 and 2 in the introduction). These intersections happen to be sectors in $\mathbb{A}$ bounded by two singular rays of same type, equivalently the union of two adjacent Weyl chambers.

Proposition 4.2. Let $x \in A_{i j}$. Then

(i) The intersection $A_{i j} \cap A_{p}$ is the sector at $y_{k}$ bounded by the rays to $p_{i}$ and $p_{j}$. That is

$$
x \in A_{p} \text { if and only if }\left\{\begin{array}{l}
\varphi_{1}(x) \geq \varphi_{1}\left(y_{k}\right) \\
\varphi_{2}(x) \leq \varphi_{2}\left(y_{k}\right)
\end{array} .\right.
$$


(ii) The intersection $A_{i j} \cap A_{D}$ is the sector at $y_{k}^{*}$ bounded by the rays to $D_{i}$ and $D_{j}$. That is,

$$
x \in A_{D} \text { if and only if }\left\{\begin{array}{l}
\varphi_{1}(x) \leq \varphi_{1}\left(y_{k}^{*}\right) \\
\varphi_{2}(x) \geq \varphi_{2}\left(y_{k}^{*}\right)
\end{array} .\right.
$$

(iii) The intersection $A_{i j} \cap A_{j k}$ is the intersection of the sector at $y_{k}$ bounded by the rays to $p_{j}$ and $D_{i} \cap D_{j}$, and the sector at $y_{k}^{*}$ bounded by the rays to $D_{j}$ and $p_{i} p_{j}$. That is,

$$
x \in A_{j k} \text { if and only if }\left\{\begin{array}{l}
\varphi_{1}(x) \geq \varphi_{1}\left(y_{k}^{*}\right) \\
\varphi_{2}(x) \geq \varphi_{2}\left(y_{k}\right) \\
\varphi_{3}(x) \leq \min \left(\varphi_{3}\left(y_{k}\right), \varphi_{3}\left(y_{k}^{*}\right)\right)
\end{array} .\right.
$$

(iv) The intersection $A_{i j} \cap A_{k i}$ is the intersection of the sector at $y_{k}$ bounded by the rays to $p_{i}$ and $D_{i} \cap D_{j}$, and the sector at $y_{k}^{*}$ bounded by the rays to $D_{i}$ and $p_{i} p_{j}$. That is,

$$
x \in A_{k i} \text { if and only if }\left\{\begin{array}{l}
\varphi_{1}(x) \leq \varphi_{1}\left(y_{k}\right) \\
\varphi_{2}(x) \leq \varphi_{2}\left(y_{k}^{*}\right) \\
\varphi_{3}(x) \geq \max \left(\varphi_{3}\left(y_{k}\right), \varphi_{3}\left(y_{k}^{*}\right)\right)
\end{array} .\right.
$$

Proof. Since $y_{k}$ is the center of the projective frame $\left(p_{i}, p_{j}, p_{i j}, p_{k}\right)$, assertion (i) comes from Proposition 2.5, as $A_{i j}=A\left(p_{i}, p_{j}, p_{i j}\right)$ and $A_{p}=A\left(p_{i}, p_{j}, p_{k}\right)$. Assertion (ii) is similar. Assertion (iii): A point $x \in A_{i j}$ lies in $A_{j k}$ if and only if, in the spherical building of directions at $\Sigma_{x} X$, the direction $\Sigma_{x} D_{j}$ is opposite to $\Sigma_{x} p_{k}$ and $\Sigma_{x} p_{j}$ is opposite to $\Sigma_{x} D_{k}$. Moreover, $\Sigma_{x} D_{j}$ is opposite to $\Sigma_{x} p_{k}$ if and only if $x \in A\left(p_{k}, p_{j}, p_{i j}\right)$. As $y_{k}$ is the center of the projective frame $\left(p_{i}, p_{j}, p_{i j}, p_{k}\right)$ and $A_{i j}=A\left(p_{i}, p_{j}, p_{i j}\right)$, the set of such $x$ is the sector at $y_{k}$ bounded by the rays to $p_{j}$ and $D_{i} \cap D_{j}$ (by Proposition 2.5). This is the subset of $x \in A_{i j}$ satisfying: $\varphi_{2}(x) \geq \varphi_{2}\left(y_{k}\right)$ and $\varphi_{3}(x) \leq \varphi_{3}\left(y_{k}\right)$. Similarly, as $y_{k}^{*}$ is the center of the projective frame $\left(D_{i}, D_{j}, D_{i j}, D_{k}\right)$ and $A_{i j}=A\left(D_{i}, D_{j}, D_{i j}\right)$, the direction $\Sigma_{x} p_{j}$ is opposite to $\Sigma_{x} D_{k}$ if and only if $x$ is in the sector at $y_{k}^{*}$ bounded by the rays to $D_{j}$ and $D_{i j}=p_{i} p_{j}$. That is, if and only if $\varphi_{1}(x) \geq \varphi_{1}\left(y_{k}^{*}\right)$ and $\varphi_{3}(x) \leq \varphi_{3}\left(y_{k}^{*}\right)$, and we are done. Assertion (iv) is similar.

In particular, as $y_{k}^{*}$ is on one of the three singular rays of type 1 issued from $y_{k}$ by Propositions 4.1, from Proposition 4.2 we easily get the following result.

Corollary 4.3. The intersections with $A_{i j}$ of $A_{j k}, A_{k i}, A_{p}$ and $A_{D}$ form a partition of $A_{i j}$.

$4 \mathrm{C}$. In the flat $A_{p}$. We now consider the flat $A_{p}=A\left(p_{1}, p_{2}, p_{3}\right)$. The following proposition describes the respective positions in $A_{p}$ of the points $y_{1}, y_{2}, y_{3}$. We identify $A_{p}$ with $\mathbb{A}$ by a marked flat $f_{p}: \mathbb{A} \rightarrow A_{p}$ sending $\partial \mathfrak{C}$ to $\left(p_{1}, p_{1} p_{2}\right)$ (hence direction $\left[\varepsilon_{i}\right]$ to $p_{i}$ for $\left.i=1,2,3\right)$. Recall that we then have $\overrightarrow{x x^{\prime}}=x^{\prime}-x=$ $B_{\left(p_{1}, p_{1} p_{2}\right)}\left(x, x^{\prime}\right)$ for $x, x^{\prime} \in A_{p}$.

Proposition 4.4. In the flat $A_{p}$ we have:

(i) In simple roots coordinates, we have $\overrightarrow{y_{2} y_{3}}=\left(z_{1}, 0\right)$.

(ii) If $z_{1} \geq 0$, the point $y_{i+1}$ is in the ray $\left[y_{i}, p_{i+2}\right)$ (for all $i$ ), and if $z_{1} \leq 0$, the point $y_{i}$ is in the ray $\left[y_{i+1}, p_{i+2}\right)$ for all $i$.

In particular the triangle $\Delta \subset A_{p}$ with vertices $y_{1}, y_{2}, y_{3}$ is singular, i.e. the sides have singular type in $\overline{\mathfrak{C}}$. 
Proof. Recall that the point $y_{k}$ is the orthogonal projection on the flat $A_{p}$ of the singular boundary point $p_{i j}=D_{i} \cap D_{j}$. Then, by Proposition 2.6 the points $y_{2}$ and $y_{3}$ have the same projection in the transverse tree $X_{p_{1}}$, that is the center of the ideal triple $\left(p_{1} p_{13}, p_{1} p_{2}, p_{1} p_{3}\right)=\left(D_{1}, p_{1} p_{2}, p_{1} p_{3}\right)=\left(p_{1} p_{23}, p_{1} p_{2}, p_{1} p_{3}\right)$, proving that $\varphi_{2}\left(\overrightarrow{y_{2} y_{3}}\right)=0$. Furthermore, by Proposition 2.9 we have

$$
\begin{aligned}
\varphi_{2}\left(\overrightarrow{y_{3} y_{1}}\right) & =\boldsymbol{\beta}\left(p_{1} p_{2}, p_{1} p_{12}, p_{1} p_{3}, p_{1} p_{23}\right) \\
& =\boldsymbol{\beta}\left(p_{1} p_{2}, D_{1}, p_{1} p_{3}, p_{1} p_{23}\right) \\
& =\boldsymbol{\beta}\left(D_{1}, p_{1} p_{2}, p_{1} p_{23}, p_{1} p_{3}\right) \\
& =z_{1}
\end{aligned}
$$

proving that $\varphi_{2}\left(\overrightarrow{y_{3} y_{1}}\right)=z_{1}$. Applying this to the permuted triple $\left(F_{3}, F_{1}, F_{2}\right)$, we obtain $\varphi_{1}\left(\overrightarrow{y_{2} y_{3}}\right)=z_{1}$ (by invariance of the geometric triple ratio $z_{1}$ by cyclic permutation). Assertion (ii) follows from (ii), applying cyclic permutations.

We now describe the intersections of $A_{p}$ with the other flats, see Figure 6.

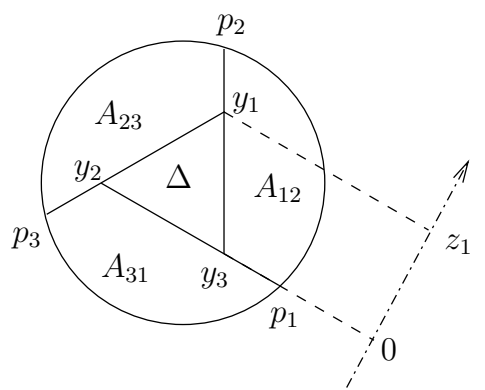

In the case $z_{1} \geq 0$.

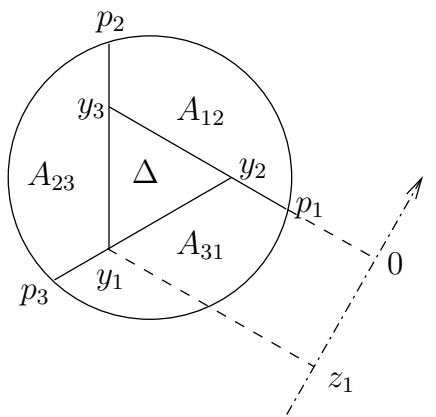

In the case $z_{1} \leq 0$.

Figure 6 . In the flat $A_{p}$.

Proposition 4.5. Let $S_{i}=A_{p} \cap A_{i, i+1}$ and let $\Delta$ be the triangle with vertices $y_{1}, y_{2}, y_{3}$. Then

(i) $S_{i}$ is the sector of $A_{p}$ bounded by the rays from $y_{i+2}$ to $p_{i}$ and $p_{i+1}$.

(ii) $S_{1}, S_{2}, S_{3}$ and $\Delta$ form a partition of $A_{p}$.

Proof. Assertion (i) follows from point (i) of Proposition 4.2. In the case where $z_{1} \geq 0$, assertion (ii) then comes from the fact that for all $i, y_{i+1}$ is in the ray $\left[y_{i}, p_{i+2}\right)$ (Proposition 4.4). The case where $z_{1} \leq 0$ is similar.

$4 \mathrm{D}$. In the flat $A_{D}$. We now state the similar properties in the dual flat $A_{D}=$ $A\left(D_{1}, D_{2}, D_{3}\right)$, which have same proofs, exchanging the role of points and lines.

Proposition 4.6. In the flat $A_{D}$ identified with $\mathbb{A}$ by a marked flat sending $\partial \mathfrak{C}$ to $\left(D_{1} \cap D_{2}, D_{1}\right)$, we have:

(i) $\overrightarrow{y_{2}^{*} y_{3}^{*}}=\left(0,-z_{1}\right)$ in simple roots coordinates. In particular $y_{2}^{*}$ and $y_{3}^{*}$ are on a common singular geodesic to $D_{1}$.

(ii) The points $y_{1}^{*}, y_{2}^{*}, y_{3}^{*}$ form a singular triangle $\Delta^{*}$ in $A_{D}$.

(iii) For all $i \in \mathbb{Z} / 3 \mathbb{Z}, S_{i}^{*}=A_{D} \cap A_{i, i+1}$ is the sector of $A_{D}$ bounded by the rays from $y_{i+2}^{*}$ to $D_{i}$ and $D_{i+1}$. 
(iv) $S_{1}^{*}, S_{2}^{*}, S_{3}^{*}$ and $\Delta^{*}$ form a partition of $A_{D}$.

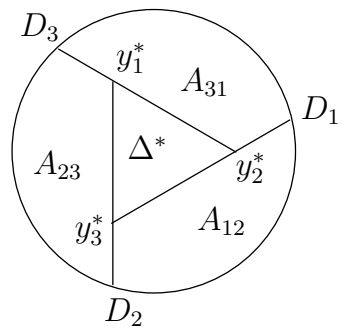

In the case $z_{1} \geq 0$.

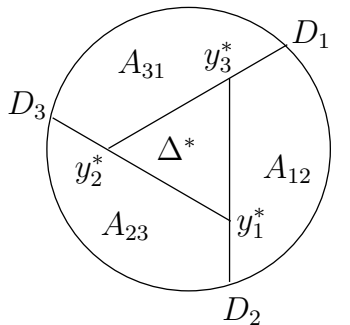

In the case $z_{1} \leq 0$.

Figure 7 . In the flat $A_{D}$.

4E. The classification. We now combine the previous results to establish the classification in two geometric types, finishing to prove Theorems 0.1 and 0.2 .

Proof of Theorem 0.1. Let $x=y_{3}$ and $x^{*}=y_{3}^{*}$. We identify the flat $A_{12}$ with the model flat $\mathbb{A}$ by a marked flat sending $\partial \mathfrak{C}$ to $F_{2}$, and 0 to $y_{3}^{*}$. By Proposition 4.2 applied to the flat $A_{12}$, we have $\varphi_{1}\left(y_{3}\right)=z_{2}, \varphi_{2}\left(y_{3}\right)=z_{3}$, and $\varphi_{3}\left(y_{3}\right)=z_{1}$. By Proposition 4.2 applied to the flat $A_{12}$, the intersection $I=A_{12} \cap A_{23} \cap A_{31}$ is the subset of $y \in A_{12}$ such that

$$
\begin{cases}0 & \leq \varphi_{1}(y) \leq \varphi_{1}\left(y_{3}\right)=z_{2} \\ 0 & \geq \varphi_{2}(y) \geq \varphi_{2}\left(y_{3}\right)=z_{3} \\ \max \left(\varphi_{3}\left(y_{3}\right), 0\right) & \leq \varphi_{3}(y) \leq \min \left(\varphi_{3}\left(y_{3}\right), 0\right) .\end{cases}
$$

In particular, if $I$ is not empty, then $z_{1}=\varphi_{3}\left(y_{3}\right)=0$.

Suppose from now on that $z_{1}=0$. Then $z_{2} \geq 0$ and $z_{3}=-z_{2}$ by the ultrametricity of the geometric triple ratio (Proposition 3.1(iv)). By the description above, $I$ is then the subset of the line $\varphi_{3}=0$ (which contains $y_{3}^{*}=0$ and $y_{3}$ ) consisting of the $y$ such that $0 \leq \varphi_{1}(y) \leq \varphi_{1}\left(y_{3}\right)$ (since $\varphi_{2}(y)=-\varphi_{1}(y)$ when $\left.\varphi_{3}(y)=0\right)$. Hence $I$ is not empty and is the segment from $0=y_{3}^{*}$ to $y_{3}$ i.e. $\left[x, x^{*}\right]$. Furthermore, as $z_{1}=0$, Proposition 4.4 implies that $y_{1}=y_{2}=y_{3}$. Similarly, we have $y_{1}^{*}=y_{2}^{*}=y_{3}^{*}$ by Proposition 4.6. Suppose now $x \neq x^{*}$. Since the segment $\left[x, x^{*}\right]$ lies in the ray $\left[x, p_{i j}\right)$, and $x=y_{k}$ is the orthogonal projection of $p_{i j}$ on $A_{p}$, we have $\varangle_{x}\left(x^{*}, D\right)=\pi$ for all lines $D$ in $\partial_{\infty} A_{p}$ (Proposition 2.4). Therefore we have $\varangle_{x}\left(x^{*}, y\right) \geq \frac{2 \pi}{3}$ for all $y \neq x$ in $A_{p}$. Similarly, we have that $\varangle_{x^{*}}(x, y) \geq \frac{2 \pi}{3}$ for all $y \neq x$ in $A_{p}$. Hence $\left[x, x^{*}\right]$ is the unique segment of minimal length joining $A_{p}$ to $A_{D}$. Assertion (iv) follows from Proposition 4.1.

Proof of Theorem 0.2. If $z_{2}>0$, then $z_{1}=0$ by the ultrametricity of the geometric triple ratio (Proposition 3.1(iv)), and $A_{p} \cap A_{D}$ is empty by Theorem 0.1. Suppose now that $z_{2} \leq 0$. Since the case $z_{1} \leq 0$ reduces to the case $z_{1} \geq 0$ by exchanging $F_{2}$ and $F_{3}$, it is enough to handle the case $z_{1} \geq 0$. Then $z_{3}=0$ and $z_{2}=-z_{1}$. Let $x_{i}=y_{i+2}$ for $i \in \mathbb{Z} / 3 \mathbb{Z}$. In $A_{i j}$ identified with $\mathbb{A}$ in such a way that $y_{k}^{*}=0$, by Proposition 4.1 we have $\varphi_{1}\left(y_{k}\right)=z_{2}=-z_{1} \leq 0, \varphi_{2}\left(y_{k}\right)=z_{3}=0$, hence $\varphi_{3}\left(y_{k}\right)=$ $z_{1} \geq 0$. By Proposition 4.2(iv), $A_{i j} \cap A_{i k}$ is the set of $x \in A_{i j} \simeq \mathbb{A}$ such that $\varphi_{1}(x) \leq \varphi_{1}\left(y_{k}\right), \varphi_{2}(x) \leq 0=\varphi_{2}\left(y_{k}\right)$ and $\varphi_{3}(x) \geq \max \left(\varphi_{3}\left(y_{k}\right), 0\right)=\varphi_{3}\left(y_{k}\right)$. This 
is the Weyl chamber $y_{k}-\overline{\mathfrak{C}}$, i.e. the Weyl chamber from $y_{k}=x_{i}$ to $F_{i}$. Similarly, $A_{i j} \cap A_{j k}$ is the Weyl chamber from $y_{k}^{*}$ to $F_{j}$. Applying a cyclic permutation $(i j k)$, i.e. working in the flat $A_{j k}$, we also similarly get that $A_{i j} \cap A_{j k}$ is the Weyl chamber from $y_{i}$ to $F_{j}$. Therefore $y_{k}^{*}=y_{i}$.

By Proposition 4.2 $A_{p} \cap A_{D} \cap A_{i j}$ is the intersection of the sector at $y_{k}^{*}$ bounded by the rays to $D_{i}$ and $D_{j}$, with the sector at $y_{k}$ bounded by the rays to $p_{i}$ and $p_{j}$. As the point $y_{k}$ is on the ray from $y_{k}$ to $D_{i}$, this is equal to the segment $\left[y_{k}, y_{k}^{*}\right]$. In particular $A_{p} \cap A_{D}$ contains $y_{k}$. Then $A_{p} \cap A_{D}$ contains $y_{1}, y_{2}$ and $y_{3}$, hence the triangle $\Delta$ with vertices $y_{1}, y_{2}$ and $y_{3}$, and since $A_{p} \cap A_{D} \cap A_{i j}=\left[y_{k}, y_{i}\right] \subset$ $\Delta$, Proposition 4.5(ii) provides the reverse inclusion. Assertion (iii) comes from Proposition 4.1.

We finally prove (iv). Let $(i, j, k)=(123)$. Looking in the flat $A_{p}$, we see that the singular triangle $\Delta$ is contained in the Weyl chamber of $X$ with tip $x_{i}$ and that at $x_{i}$, we have $\Sigma_{x_{i}} x_{j}=\Sigma_{x_{i}} p_{j}$. Looking in the flat $A_{D}$ we get $\Sigma_{x_{i}} x_{k}=\Sigma_{x_{i}} D_{k}$. Hence $\Sigma_{x_{i}} \Delta=\left(\Sigma_{x_{i}} p_{j}, \Sigma_{x_{i}} D_{k}\right)$. Since $x_{i}$ belongs to the flats $A\left(F_{i}, F_{j}\right)$ and $A\left(F_{i}, F_{k}\right)$, we have that $\Sigma_{x_{i}} p_{j}$ is opposite to $\Sigma_{x_{i}} D_{i}$ and that $\Sigma_{x_{i}} D_{k}$ is opposite to $\Sigma_{x_{i}} p_{i}$. Therefore the Weyl chambers $\Sigma_{x_{i}} \Delta$ and $\Sigma_{x_{i}} F_{i}$ are opposite. It implies that $\Delta$ and the Weyl chamber from $x_{i}$ to $F_{i}$ are contained in a common flat of $X$ by basic properties of real Euclidean buildings (see property (CO) of [Par00]).

In the algebraic case the following remark provides an alternative proof of some of the assertions of Theorem 0.2 .

Remark 4.7. Let $\tilde{p}_{i}$ in $V=\mathbb{K}^{3}$ be a vector representing $p_{i}$ and $\tilde{D}_{i}$ in $V^{*}$ be a linear form representing $D_{i}$. Let $\mathbf{v}=\left(v_{1}, v_{2}, v_{3}\right)$ be the basis of $V$ dual to the basis $\left(\tilde{D}_{1}, \tilde{D}_{2}, \tilde{D}_{3}\right)$ of $V^{*}$. Then in the projective plane $\left[v_{i}\right]=D_{j} \cap D_{k}$. We may suppose that $\tilde{p}_{1}=(0,1,1), \tilde{p}_{2}=(Z, 0,1), \tilde{p}_{3}=(1,1,0)$ in the basis $\mathbf{v}$, with $Z=\operatorname{Tri}\left(F_{1}, F_{2}, F_{3}\right)$. Then the element $g \in \mathrm{GL}(V)$ with matrix in the basis $\mathbf{v}$

$$
\left(\begin{array}{ccc}
1 & 1 & 0 \\
0 & 1 & 1 \\
1 / Z & 0 & 1
\end{array}\right)
$$

sends $\left[v_{i}\right]$ to $p_{i+1}$, hence $A_{D}$ to $A_{p}$. If $|1+Z| \geq 1$ and $z=\log |Z| \geq 0$, then the fixed point set of $g$ in $A_{D}$ is the image by the marked flat $f_{\mathbf{v}}$ of the singular triangle $\left\{\lambda \in \overline{\mathfrak{C}}\left|\lambda_{1}-\lambda_{3} \leq \log \right| Z \mid\right\}$ (that is, $\Delta$ ).

$4 \mathrm{~F}$. Complements. We add here for future use a simple description of the vertices $x_{i}, x_{j}, x_{k}$ of the singular triangle $\Delta$ in Theorem 0.2 by the projections on transverse trees at infinity.

Lemma 4.8. Under the hypotheses and notations of Theorem 0.2, we have the following properties.

(i) The projection $\pi_{p_{i}}\left(x_{i}\right)$ of $x_{i}$ on the tree $X_{p_{i}}$ is the center of the ideal tripod $\left(D_{i}, p_{i} p_{j}, p_{i} p_{k}\right)$.

(ii) The projection $\pi_{D_{i}}\left(x_{i}\right)$ of $x_{i}$ on the tree $X_{D_{i}}$ is the center of the ideal tripod $\left(p_{i}, D_{i} \cap D_{j}, D_{i} \cap D_{k}\right)$.

(iii) The projection $\pi_{p_{i}}\left(x_{j}\right)$ is the center of the ideal tripod $\left(D_{i}, p_{i} p_{j}, p_{i} p_{j k}\right)$.

(iv) The projection $\pi_{D_{i}}\left(x_{j}\right)$ is the center of the ideal tripod $\left(p_{i}, D_{i} \cap D_{j}, D_{i} \cap\right.$ $\left.D_{j k}\right)$.

Proof. As the point $x_{i}$ belongs to the three flats $A\left(F_{k}, F_{i}\right)$ and $A\left(F_{j}, F_{i}\right)$ and $A\left(p_{i}, p_{j}, p_{k}\right)$, its projection in the tree $X_{p_{i}}$ belongs to the projection of $A\left(F_{j}, F_{i}\right)$, 
which is the line from $D_{i}$ to $p_{i} p_{j}$, to the projection of $A\left(F_{k}, F_{i}\right)$, which is the line from $D_{i}$ to $p_{i} p_{k}$, and to the projection of $A\left(p_{i}, p_{j}, p_{k}\right)$, which is the line from $p_{i} p_{j}$ to $p_{i} p_{k}$. Hence (i) is proven. Assertion (ii) is proven in the same way.

We now prove (iii). By (ii) applied to $x_{j}$, we have that $\pi_{D_{j}}\left(x_{j}\right)$ is the center of the ideal tripod $p_{j}, p_{j k}=D_{j} \cap D_{k}, D_{j} \cap D_{i}$. As $x_{j}$ is on a geodesic from $D_{j}$ to $p_{i}$, we may deduce that $\pi_{p_{i}}\left(x_{j}\right)$ is the center of the ideal tripod $p_{i} p_{j}, p_{i} p_{j k}, D_{i}$ (using the canonical isomorphim $X_{D_{j}} \stackrel{\sim}{\rightarrow} X_{p_{i}}$ ). The last assertion (iv) has identical proof.

\section{REFERENCES}

[Bal08] Andreas Balser. Convex rank 1 subsets of Euclidean buildings (of type $A_{2}$ ). Geom. Dedicata, 131:123-158, 2008.

[BH99] Martin R. Bridson and André Haefliger. Metric spaces of non-positive curvature, volume 319 of Grundlehren der Mathematischen Wissenschaften. Springer-Verlag, Berlin, 1999.

[BIW10] M. Burger, A. Iozzi, and A. Wienhard. Surface group representations with maximal Toledo invariant. Ann. of Math. (2), 172:517-566, 2010.

[Bou96] Marc Bourdon. Sur le birapport au bord des CAT(-1)-espaces. Publ. Math., Inst. Hautes Étud. Sci., 83:95-104, 1996.

[BT84] François Bruhat and Jacques Tits. Schémas en groupes et immeubles des groupes classiques sur un corps local. Bull. Soc. Math. Fr., 112:259-301, 1984.

[CN06] Jean-Louis Clerc and Karl-Hermann Neeb. Orbits of triples in the Shilov boundary of a bounded symmetric domain. Transform. Groups, 11:387-426, 2006.

[FG06] Vladimir Fock and Alexander Goncharov. Moduli spaces of local systems and higher Teichmüller theory. Publ. Math., Inst. Hautes Étud. Sci., 103:1-211, 2006.

[FG07] V.V. Fock and A.B. Goncharov. Moduli spaces of convex projective structures on surfaces. Adv. Math., 208:249-273, 2007.

[GI63] O. Goldman and N. Iwahori. The space of p-adic norms. Acta Math., 109:137-177, 1963.

[Gol80] W. M. Goldman. Discontinuous groups and the Euler class. PhD thesis, University of California, Berkeley, 1980.

[Gol99] William M. Goldman. Complex hyperbolic geometry. Oxford: Clarendon Press, 1999.

[KL97] B. Kleiner and B. Leeb. Rigidity of quasi-isometries for symmetric spaces and Euclidean buildings. Publ. Math., Inst. Hautes Étud. Sci., 86:115-197 (1998), 1997.

[Lee00] Bernhard Leeb. A characterization of irreducible symmetric spaces and Euclidean buildings of higher rank by their asymptotic geometry, volume 326 of Bonner Mathematische Schriften. Universität Bonn Mathematisches Institut, Bonn, 2000.

[MSv14] Bernhard Mühlherr, Koen Struyve, and Hendrik van Maldeghem. Descent of affine buildings. I: Large minimal angles. Trans. Am. Math. Soc., 366:4345-4366, 2014.

[Ota92] Jean-Pierre Otal. Sur la géométrie symplectique de l'espace des géodésiques d'une variété à courbure négative. Rev. Mat. Iberoam., 8:441-456, 1992.

[Par00] A. Parreau. Immeubles affines: construction par les normes et étude des isométries. In Crystallographic groups and their generalizations (Kortrijk, 1999), volume 262 of Contemp. Math., pages 263-302. Amer. Math. Soc., 2000.

[Par11] A. Parreau. Espaces de représentations complètement réductibles. J. Lond. Math. Soc., II. Ser., 83:545-562, 2011.

[Par12] A. Parreau. Compactification d'espaces de représentations de groupes de type fini. Math. Z., 272:51-86, 2012.

[Par15] A. Parreau. Invariant subspaces for some surface groups acting on $A_{2}$-Euclidean buildings. ArXiv e-prints, April 2015.

[Rou09] Guy Rousseau. Euclidean buildings. In Géométries à courbure négative ou nulle, groupes discrets et rigidités, volume 18 of Sémin. Congr., pages 77-116. Soc. Math. France, 2009.

[Tal06] Malik Talbi. A Haagerup inequality, deformation of triangles and affine buildings. J. Inst. Math. Jussieu, 5:187-227, 2006.

[Tit74] Jacques Tits. Buildings of spherical type and finite BN-pairs, volume 386 of Lecture Notes in Math. Springer-Verlag, 1974. 
[Tit86] Jacques Tits. Immeubles de type affine. In Buildings and the geometry of diagrams (Como, 1984), volume 1181 of Lecture Notes in Math., pages 159-190. Springer-Verlag, 1986.

[Tol89] Domingo Toledo. Representations of surface groups in complex hyperbolic space. J. Differ. Geom., 29:125-133, 1989.

Université Grenoble Alpes et CNRS, Institut Fourier, Grenoble, France

E-mail address: Anne.Parreau@univ-grenoble-alpes.fr 